\title{
Bias Correction, Anonymization, and Analysis of Smartphone Pressure Observations Using Machine Learning and Multiresolution Kriging
}

\author{
CALlie MCNichOlas ${ }^{\mathrm{a}}$ AND ClifFORD F. MASs ${ }^{\mathrm{a}}$ \\ ${ }^{a}$ Department of Atmospheric Sciences, University of Washington, Seattle, Washington
}

(Manuscript received 24 November 2020, in final form 24 June 2021)

\begin{abstract}
With over a billion smartphones capable of measuring atmospheric pressure, a global mesoscale surface pressure network based on smartphone pressure sensors may be possible if key technical issues are solved, including collection technology, privacy, and bias correction. To overcome these challenges, a novel framework was developed for the anonymization and bias correction of smartphone pressure observations (SPOs) and was applied to billions of SPOs from the Weather Company (IBM). Bias correction using machine learning reduced the errors of anonymous (ANON) SPOs and uniquely identifiable (UID) SPOs by $43 \%$ and 57\%, respectively. Applying multiresolution kriging, gridded analyses of bias-corrected smartphone pressure observations were made for an entire year (2018), using both anonymized (ANON) and nonanonymized (UID) observations. Pressure analyses were also generated using conventional Meteorological Assimilation Data Ingest System (MADIS) surface pressure networks. Relative to MADIS analyses, ANON and UID smartphone analyses reduced domain-average pressure errors by $21 \%$ and $31 \%$, respectively. The performance of smartphone and MADIS pressure analyses was evaluated for two high-impact weather events: the landfall of Hurricane Michael and a long-lived mesoscale convective system. For these two events, both anonymized and nonanonymized smartphone pressure analyses better captured the spatial structure and temporal evolution of mesoscale pressure features than the MADIS analyses.
\end{abstract}

SIGNIFICANCE STATEMENT: Smartphones offer the potential to vastly expand the coverage and density of surface pressure observations available to weather models. To date, privacy concerns and poor data quality have limited the utility of smartphone pressure observations (SPOs). In this study, we address these issues by developing a method for anonymizing and bias correcting the SPOs collected by IBM's Weather Channel smartphone app. Importantly, anonymizing SPOs did not substantially degrade their utility. Pressure analyses produced with anonymous and identified SPOs outperformed pressure analyses from existing surface pressure networks. During a major hurricane, smartphone pressure analyses did a better job capturing the observed surface pressure minimum than analyses based on current networks.

KEYWORDS: Atmosphere; Hurricanes; Mesoscale systems; Pressure; Surface pressure; Automatic weather stations; Data processing; Data quality control; In situ atmospheric observations; Surface observations; Bias; Error analysis; Interpolation schemes; Kalman filters; Numerical analysis/modeling; Quality assurance/control; Statistical techniques; Data science; Decision trees; Machine learning

\section{Introduction}

Many operational centers around the world run limitedarea weather models at convection-allowing grid spacing (Alexander et al. 2017; Gustafsson et al. 2018), reflecting a steady improvement in the physics and resolution of numerical weather prediction (NWP) (Bauer et al. 2015). A key advance underlying this improvement is the successful incorporation of large amounts of observational data from satellites, mesoscale networks, and weather radars to initialize numerical model forecasts (Simmons and Hollingsworth 2002; Benjamin et al. 2019). As NWP systems progressively move to convection-allowing resolution, improvements in forecast accuracy will depend on the availability of densely spaced mesoscale observations (Gustafsson et al. 2018) to produce initial conditions through data assimilation (Liu and Rabier 2002) and for verification. The value of surface pressure data is particularly large because it can be used to retrieve the three-dimensional atmospheric structure.

\footnotetext{
Corresponding author: Callie McNicholas, cmcnich@uw.edu
}

NWP models have applied in situ pressure observations for verification, data assimilation, and reanalysis (Dirren et al. 2007; Benjamin et al. 2010; Compo et al. 2006). Modern NWP systems assimilate such surface pressure observations from a global network of $\sim 5000$ METAR and $\sim 10000$ synoptic (SYNOP) stations, at frequencies ranging from subhourly to six hourly (Haiden et al. 2018). While these observations have benefited NWP (Ingleby 2015; Chang et al. 2017), their synoptic distribution and temporal availability limit their utility for determining mesoscale structures and thus mesoscale modeling (Sun et al. 2014; Wheatley and Stensrud 2010).

To satisfy the increasing demand for additional observation density in support of global high-resolution NWP, several studies have examined whether NWP can benefit from personal weather station (PWS) observations (Madaus et al. 2014; Sobash and Stensrud 2015; Gasperoni et al. 2018; Nipen et al. 2020; Droste et al. 2020; Mandement and Caumont 2020). Their findings suggest that with robust quality control and bias correction (Miller et al. 2005; Meier et al. 2017; Napoly et al. 2018; Clark et al. 2018), PWS observations can reduce NWP forecast error, especially in regions sparsely observed by 
standard synoptic networks (Nipen et al. 2020; Mandement and Caumont 2020). While PWS networks increase in size throughout North America (Miller et al. 2005) and Europe (Clark et al. 2018), they are generally restricted to a weather enthusiasts and are typically sparse in regions of low population density. The cost and infrastructure requirements of PWSs also impede their growth, especially in low- to middleincome countries. Furthermore, the instrumentation quality of PWS varies considerably and there are often no instrument siting standards.

Given the limitations of current surface weather observing networks, there has been increasing interest in acquiring meteorological data through crowdsourced observations (Muller et al. 2015; Zheng et al. 2018). In most cases, crowdsourcing involves the retrieval of data from internet-connected devices (Muller et al. 2015; Arthur et al. 2018) that are typically not designed for weather monitoring.

One of the most promising sources of crowdsourced meteorological data is smartphones. Since 2012, manufacturers have produced smartphones containing pressure sensors (Mass and Madaus 2014), and today a billion smartphones can measure atmospheric pressure. Smartphones are available worldwide, can measure accurate horizontal positions, and include communication infrastructure in the form of a mobile broadband (internet) connection. Smartphone penetration is increasing fastest in regions, such as sub-Saharan Africa (Ericsson 2020), where additional surface observations are most needed (WMO 2014). Although pressure is currently the only atmospheric measurement available from most smartphones (McNicholas and Mass 2018a), the assimilation of surface pressure has a greater impact on global NWP than the assimilation of other surface variables (Rabier et al. 2007; Ingleby 2015). Accordingly, if smartphone pressure observations (SPOs) can be retrieved with sufficient quality and quantity, they could have a positive impact on weather forecasting.

Initial efforts to acquire, perform quality control (QC) on, and apply smartphone pressure observations have been encouraging (Kim et al. 2015; Kim et al. 2016; Madaus and Mass 2017). McNicholas and Mass (2018a) demonstrated that SPOs from thousands of smartphones across the Pacific Northwest could be retrieved in real time with subhourly frequency. A machine learning bias-correction approach was developed wherein random forests were trained to predict SPO errors using solely smartphone sensor data. Remarkably, approximately $84 \%$ of SPOs were retained after bias correction and QC. In comparison, Hintz et al. (2019a) used a simplified approach to bias correction wherein NWP model analyses were used to compute a single bias estimate for each unique smartphone. In their study, only $12 \%$ of SPOs were retained after bias correction and QC.

Experiments assimilating SPOs in convection-allowing NWP have produced mixed results. When SPO bias correction and QC were dependent on contemporaneous observations from other surface pressure networks for calibration and QC, the assimilation of SPOs had a mixed (Madaus and Mass 2017) or negative impact on short-term forecast error (Hintz et al. 2019a). In contrast, when SPO bias correction and QC was not dependent on contemporaneous observations from other surface pressure networks, the assimilation of SPOs improved short-term forecast skill (McNicholas and Mass 2018b). Before broader conclusions can be drawn about the utility of SPOs for NWP, the impacts of expanding the availability of smartphone observations from the thousands to millions must be evaluated. Fortunately, this task is now feasible thanks to IBM (the Weather Company), which has provided access to SPOs from smartphones running on its flagship Weather Channel mobile app.

Building on the work of McNicholas and Mass (2018a), this paper presents an improved methodology for bias correction of smartphone pressures that is capable of scaling to billions of SPOs. In addition, a technique is proposed and tested for the anonymous retrieval and bias correction of SPOs. Using these techniques, SPOs retrieved over the central and eastern United States are bias corrected over an entire year, and the resulting datasets are used to examine the following questions:

1) Can machine learning be used to effectively bias correct large numbers of smartphone pressure observations?

2) To what degree is the quality of SPOs reduced when bias correction is performed anonymously?

3) Can SPOs produce better pressure analyses than possible using current surface pressure networks?

\section{Pressure datasets}

This study makes use of pressure observations from the Meteorological Assimilation Data Ingest System (MADIS; Miller et al. 2005) and a smartphone pressure dataset from the Weather Company (IBM), both retrieved between 15 August 2017 and 30 December 2018. In this study, pressure datasets were retrieved across the central and eastern United States. The western United States was excluded due to the relatively low numbers of smartphone pressures collected in the region by IBM; furthermore, MADIS observations, used for calibration, are relatively sparse across the Intermountain West.

\section{a. MADIS pressure dataset}

MADIS provides sea level pressure (i.e., altimeter setting) observations from local mesonets and METARs. The majority of MADIS mesonet observations were provided by the Citizen Weather Observer Program (CWOP; www.wxqa.com), a network of PWSs maintained by private citizens. Mesonet observations generally report pressure to $0.01 \mathrm{hPa}$ while METARs, provided by MADIS, report pressure to the nearest 0.01 inch of mercury $(\sim 0.33 \mathrm{hPa})$. To address the lower resolution of MADIS pressure observation at METAR locations, 1-min station pressure observations with a resolution of $\sim 0.03 \mathrm{hPa}$ were retrieved from the Automated Surface Observing System (ASOS) archive (NCEI 2018). Following Eq. (2) of McNicholas and Mass (2018a), a 5-min station pressure average was computed and reduced to sea level assuming the U.S. Standard Atmosphere, 1976 (COESA 1976), thus being equivalent to altimeter setting.

MADIS pressure observations undergo three levels of QC. A validity check evaluates whether pressure observations are within a physically reasonable range (NCEP 2017). For temporal consistency, pressure change is calculated to determine if a reasonable threshold is exceeded. Last, a spatial-consistency (buddy) check compares pressure observations to neighboring 
TABLE 1. Smartphone metadata provided by IBM (WC).

\begin{tabular}{ll}
\hline \multicolumn{1}{c}{ Variable name } & \multicolumn{1}{c}{ Description } \\
\hline $\begin{array}{l}\text { Unique identifier } \\
\text { Latitude }\end{array}$ & $\begin{array}{l}\text { Hashed device ID } \\
\text { The north-south position of the smartphone } \\
\text { on Earth's surface } \\
\text { The east-west position of the smartphone on } \\
\text { Earth's surface }\end{array}$ \\
Pressure & $\begin{array}{l}\text { Ambient pressure from the smartphone } \\
\text { microelectromechanical sensor. }\end{array}$ \\
$\begin{array}{l}\text { Horizontal location } \\
\text { accuracy }\end{array}$ & $\begin{array}{l}\text { The radius of a circle centered at the device's } \\
\text { latide and longitude. The probability that } \\
\text { the device lies within this circle is } 68 \% \text {. The } \\
\text { horizontal accuracy is thus a radius of } 68 \% \\
\text { confidence. }\end{array}$ \\
\hline
\end{tabular}

observations through optimum interpolation and cross validation (Miller et al. 2005). Since METAR observations from the ASOS archive were not quality controlled, the MADIS spatial consistency check was applied to these observations. On average, less than $1 \%$ of ASOS pressure observations were flagged by this QC check.

\section{b. Smartphone pressure dataset}

All SPOs used in this study were provided by IBM and were retrieved from smartphones through the Weather Channel (WC) app. A list of variables retrieved from each smartphone is provided in Table 1. A unique identifier (UID) was collected from each smartphone that facilitated bias correction. A USGS digital elevation map (DEM) was used to extract the ground elevation at the location of each smartphone (Gesch et al. 2014). Elevation uncertainty was computed by calculating two standard deviations of the nine DEM points closest to the smartphone. Altimeter setting was calculated using the DEM ground elevation following the approach of McNicholas and Mass (2018a).

While the assumption that smartphones are at ground level is often inaccurate, assigning the ground elevation at the location of the SPO provides a consistent first guess. Using a DEM to estimate elevation guarantees that for a given latitude and longitude the same elevation will always be retrieved. This is not the case with GPS altitude, which is notoriously inaccurate and can vary depending on battery life, time of day, and location. Furthermore, GPS altitude is often not available indoors. Using a DEM thus ensures an elevation estimate is always available and consistent.

\section{c. Temporal characteristics of smartphone pressures}

Unlike conventional pressure observations, SPOs are retrieved irregularly. Boxplots in Fig. 1 display the distribution of pressure observation frequency for each observing platform: METARs, mesonets, and smartphones. The median observation frequency for SPOs was approximately once per day. In contrast, the median observation frequency for METARs and mesonet stations was approximately 10-20 times per hour, respectively. Most SPOs collected by IBM were retrieved only when the user was actively using the WC app. In general, SPOs are collected by that app when the user moves a significant distance or sufficient time has elapsed to trigger a weather update (P. Neilley, IBM, 2020, personal communication).
Figure 1c shows the number of SPOs available within a 5-min window, averaged by the time of day, throughout the study. SPO counts vary diurnally, peaking early in the morning and late in the afternoon. At these times, users are often on the move and thus more likely to contribute SPOs. The availability of SPOs drops significantly overnight, reaching a minimum at approximately 0800 UTC. This pattern of diurnal variation in SPO counts is similar to that found by McNicholas and Mass (2018a). Compared to SPOs, hourly observation counts from mesonets and METARs did not vary throughout the day. There is a subhourly cycle in mesonet observation counts since stations contribute observations at fixed intervals ranging from $5 \mathrm{~min}$ to $1 \mathrm{~h}$.

\section{d. Spatial characteristics}

The majority of SPOs from the Weather Channel app were retrieved over the central and eastern United States, which also possesses substantial mesonet and METAR assets and relatively dense station spacing. Figure 2 shows the distance from each smartphone observation to the nearest MADIS surface pressure observation. Over a 5-min window, the average distance to the nearest MADIS station was approximately $29 \mathrm{~km}$ (Fig. 2a). Over the same 5-min period, the average distance to the nearest SPO was around $5.5 \mathrm{~km}$ (Fig. 2b). When the observation window was extended to an entire day, the distribution of MADIS observations did not change significantly since METAR and mesonet stations have fixed locations and observing times (Fig. 2c). Over a full day, the spatial density of SPOs doubled, with an SPO located within $2.5 \mathrm{~km}$ of half of each other across the central and eastern United States (Fig. 2d). Since smartphones are mobile, a single smartphone can provide multiple observations at different locations over a day.

\section{Anonymization}

The collection of SPOs from smartphones raises issues of data privacy. Since SPOs are usually associated with location data, their retrieval must adhere to regulations such as the General Data Protection Regulation (European Union 2018) ${ }^{1}$ and the California Consumer Privacy Act (State of California 2020). ${ }^{2}$ Several previous smartphone assimilation studies have not considered privacy issues, using UIDs for SPO bias

\footnotetext{
${ }^{1}$ The GDPR (https://gdpr.eu/what-is-gdpr/) applies to anyone (including those outside the European Union) who processes the personal data of EU citizens or residents. The GDPR defines personal data more as any information that relates to an individual who can be directly or indirectly identified. This notably includes location information.

${ }^{2}$ The CCPA (https://oag.ca.gov/privacy/ccpa) provides privacy rights only to residents of California. The law applies to for-profit businesses that do business in California and have a gross annual revenue over $\$ 25$ million and buy, receive, or sell the personal information of 50000 or more California residents, households, or devices or derive $50 \%$ or more of their annual revenue from selling California residents' personal information. The CCPA defines personal information as information that identifies, relates to, or could reasonably be linked with you or your household. Under this definition, geolocation data are considered personal information.
} 
a) METAR

5-min Ob Count by Time of Day

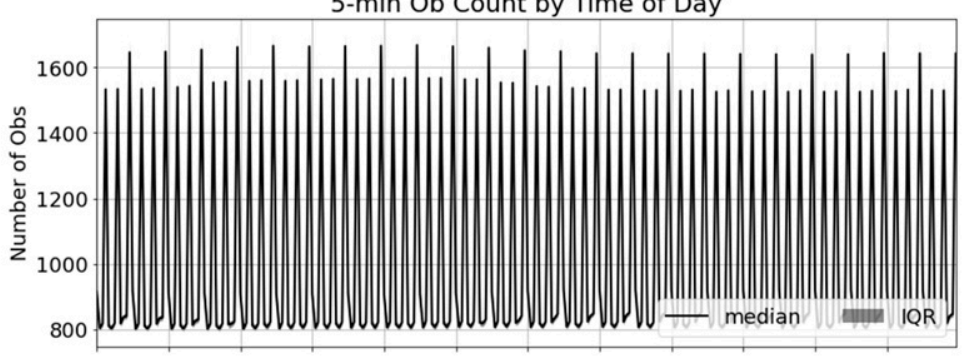

b) Mesonet

5-min Ob Count by Time of Day

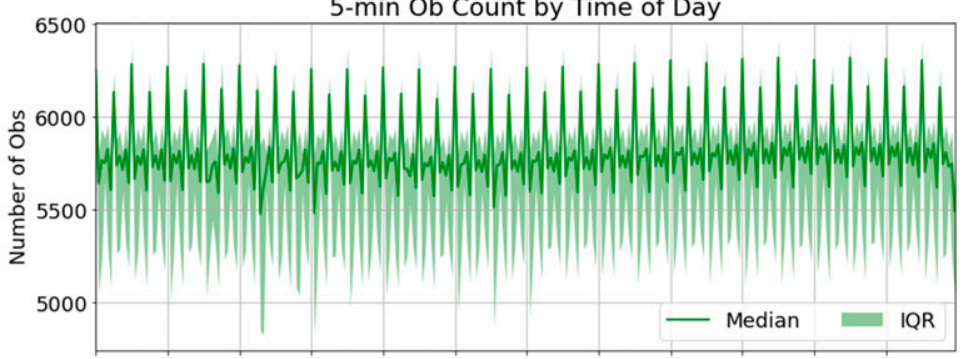

c) Smartphone

5-min Ob Count by Time of Day

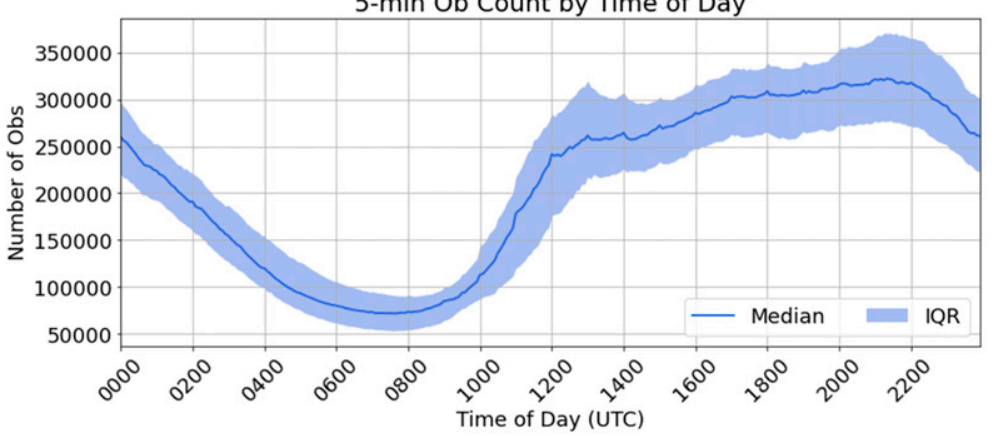

METAR

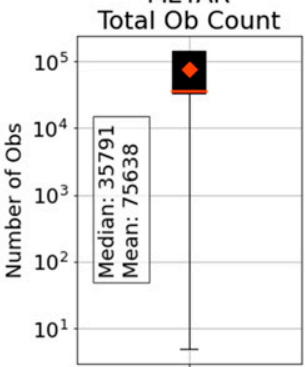

Mesonet

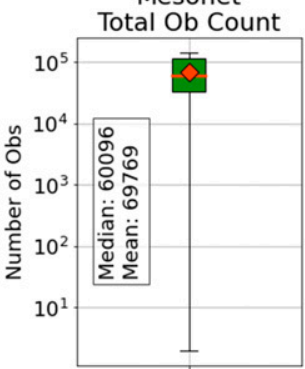

Total Ob Count

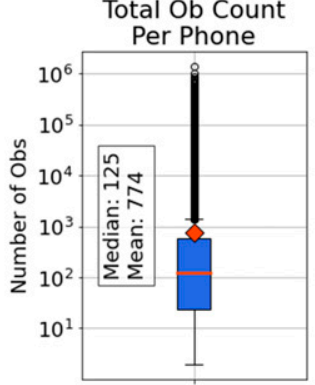

METAR

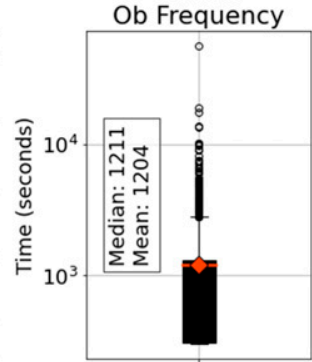

Mesonet

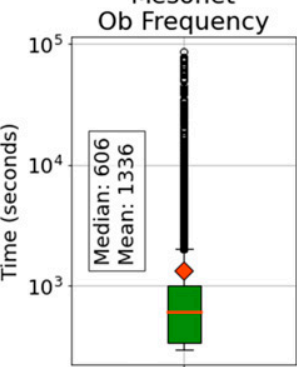

Ob Frequency Per Phone

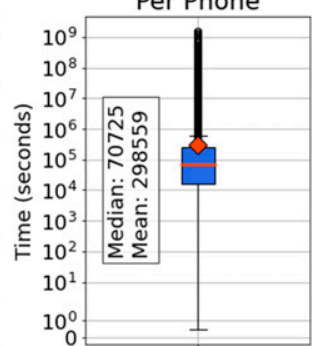

FIG. 1. 5-min observation counts for (a) METAR, (b) mesonet, and (c) smartphone datasets. The solid lines in the left panels depict the median 5-min observation count as a function of the time of day. The shaded area indicates the interquartile range (IQR). Observation counts were averaged by the time of day, every $5 \mathrm{~min}$, between 15 Aug 2017 and $30 \mathrm{Dec} 2018$. The first line of boxplots displays the distribution of the total number of observations retrieved from each station or unique smartphone. The rightmost boxplots show the distribution of average observation frequency, for each station or unique smartphone. The orange line bisecting each boxplot represents the median of the distribution, while the orange diamond depicts the mean of the distribution.

correction (McNicholas and Mass 2018a; Hintz et al. 2019a), but that approach will not be viable for future operational application (Hintz et al. 2019b). To ameliorate privacy concerns, this work has developed a framework to anonymize SPOs by modifying or removing personally identifiable information. This transformation is accomplished by combining two state-of-the-art privacy models: $k$-anonymity (Samarati and Sweeny 1998) and geo-indistinguishability (Andrés et al. 2013).

In $k$-anonymity, the prevailing privacy model for geospatial data in the literature (Gruteser and Grunwald 2003; Zandbergen 2014; Wang and Kwan 2020), a smartphone's location is hidden within a spatiotemporal region where there are $k-1$ other potential individuals (Krumm 2007; Bamba et al. 2008; Wang and Kwan 2020; Kraemer et al. 2020). The $k$-anonymity is estimated by computing the number of potential individuals in the spatiotemporal region (appendix B). Under this framework, the probability of an adversary without external knowledge identifying an individual is at most $1 / k$. In contrast to $k$-anonymity, geo-indistinguishability modifies a smartphone's location by adding controlled random noise (Andrés et al. 2013). For SPOs, personally identifiable information consists of location data and a UID. Even when UIDs are not provided, an individual's location history can reveal personally identifiable information (De Montjoye et al. 2013; Li and Goodchild 2013; Boukoros et al. 2019). This is notable as parallel efforts to anonymize SPOs in China ( $\mathrm{Li}$ et al. 2021) failed to recognize that location data are inherently personally identifiable. SPO anonymization requires the removal of the UID (Table 1) and the modification of location data.

In this study, SPO location data were modified, under the notion of geo-indistinguishability, using the planar Laplace mechanism (Bordenabe 2014). The resulting mechanism satisfies $\epsilon$-geo-indistinguishability where $\epsilon=l / r$. In this work, the privacy level $l$ and radius $r$ were set to $2 \mathrm{~km}^{-1}$ and $0.5 \mathrm{~km}$, 


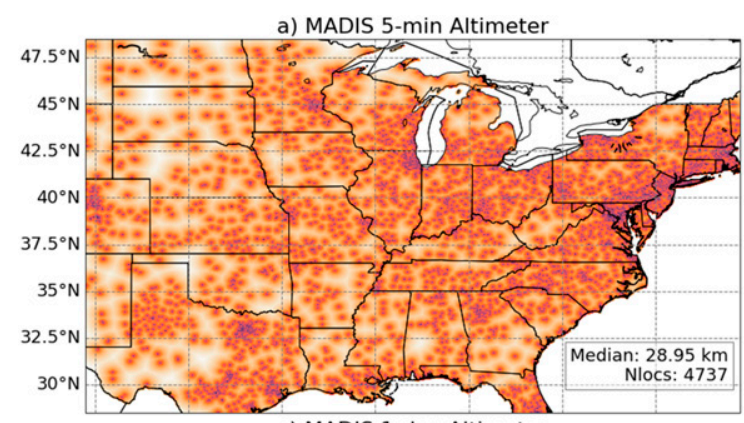

c) MADIS 1-day Altimeter
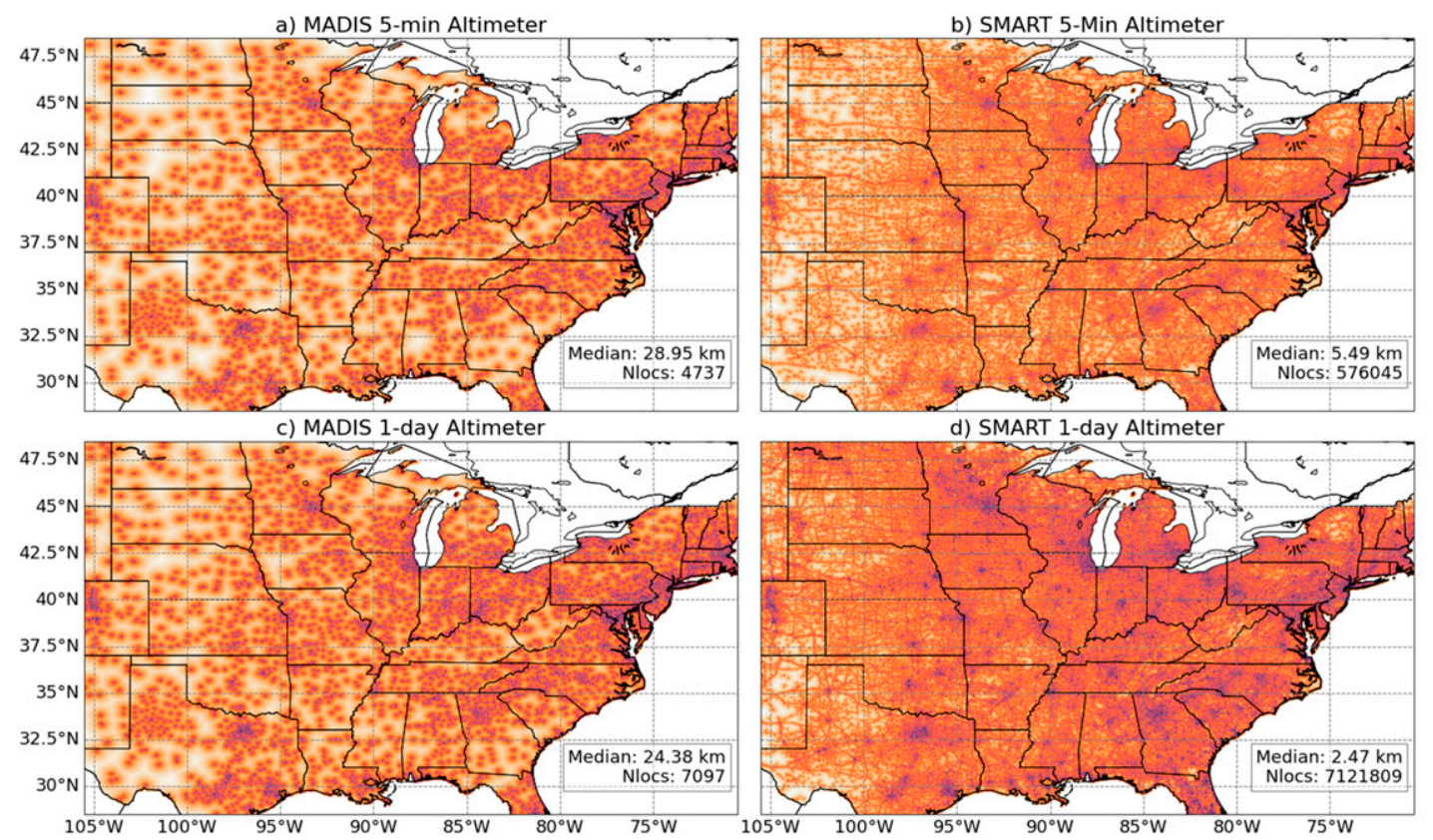

d) SMART 1-day Altimeter
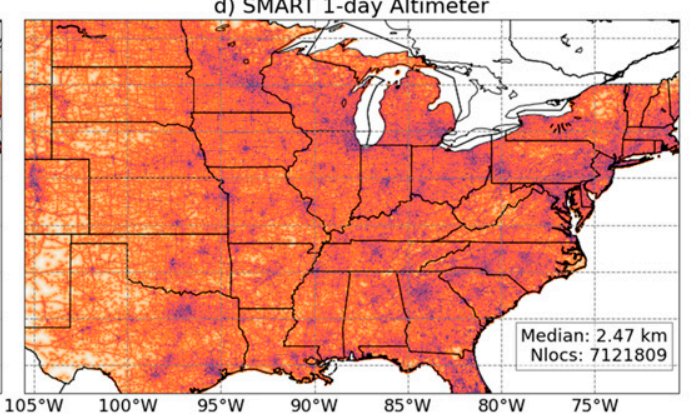

FIG. 2. The (minimum) distance to the nearest surface pressure observation at locations across the central and eastern United States. Distribution of the distance to the nearest (a) MADIS and (b) smartphone pressure observation during a 5-min window centered at 0000 UTC 1 Jul 2018. (c),(d) As in (a) and (b), but calculated from observations retrieved over a 24-h period between 0000 UTC 1 Jul and 0000 UTC 2 Jul 2018. In all four plots, darker colors represent locations where the surface observation density is relatively high and the distance to the nearest pressure observation is small. "Nlocs" is the number of unique observation locations used to generate each plot.

respectively (Bordenabe 2014). These values provide reasonable privacy protection against localization attacks (Mendes et al. 2020) and fall within common bounds in the literature (Andrés et al. 2013; Chatzikokolakis et al. 2015). With a radius $r=0.5 \mathrm{~km}$, geo-indistinguishability protects a user's location by ensuring obfuscated locations are statistically indistinguishable from others within that radius. Specifically, bivariate Laplacian noise (Bordenabe 2014) was added to obfuscate the observed location of SPOs. Subsequently, the principle of $k$-anonymity was applied to obfuscated SPO locations by rounding (or generalizing) location coordinates (Krumm 2007) to the nearest $0.25^{\circ}(\sim 25 \mathrm{~km})$. In this work, SPO anonymization was performed remotely; however, this approach requires the retrieval and storage of precise location data and thus has privacy issues. A potential solution to this problem is proposed in appendix A.

The process of anonymization is visualized in Fig. 3. Obfuscating locations through geo-indistinguishability results in a distribution of SPOs that is, by definition, very similar to the true spatial distribution of SPOs (Figs. 3a,b). Over longer periods (Figs. 3c,d) the privacy guarantees of geo-indistinguishability decline (Niu et al. 2021) as the spatial distribution of obfuscated SPO locations centers around exact locations frequented by SPOs (e.g., homes, workplaces). Due to location obfuscation, some SPO locations were rounded to a different grid point than they would have if their exact location had been used instead. Consequently, even if a single SPO falls within a $0.25^{\circ}$ grid box during a 5-min period, an adversary cannot be certain that the smartphone contributing the SPO is located within the grid box (Figs. 3e,f). Generalizing obfuscated SPOs, therefore, offers additional privacy protections beyond that afforded by $k$-anonymity alone (Fung et al. 2010). A demonstration of this anonymization approach has been made available via GitHub (https://github.com/cmac994/spo_anon).

Using gridded population counts (Columbia University 2018) and annual average traffic data (Federal Highway Administration 2017) estimates of $k$-anonymity were derived for anonymized SPOs (see appendix B). Figure 4a, shows the spatial distribution of the minimum $k$-anonymity, over a $24-h$ period, at generalized SPO locations across the central and eastern United States. Throughout most of the region, where observational density is large, $k$-anonymity is great, especially around urban centers and along interstate highways. In Fig. 4b, a boxplot shows the distribution of $k$-anonymity for all anonymous SPOs collected during May 2018. During this period, the median $k$-anonymity exceeded 10000 . In this study, anonymous SPOs were removed from the dataset if they fell within grid boxes where the minimum $k$-anonymity was below 100 .

\section{Methodology}

Figure 5 outlines the SPO bias correction and QC approach described below. MADIS pressure analyses were used to estimate SPO errors for both anonymized (ANON) and UID SPOs. Machine learning was then applied to correct SPO errors for new observations, and quality control procedures were developed to remove outlier SPOs after bias correction. Smartphone pressure analyses were subsequently generated with bias-corrected and QC'd SPOs, using the kriging technique, for comparison with MADIS pressure analyses. 


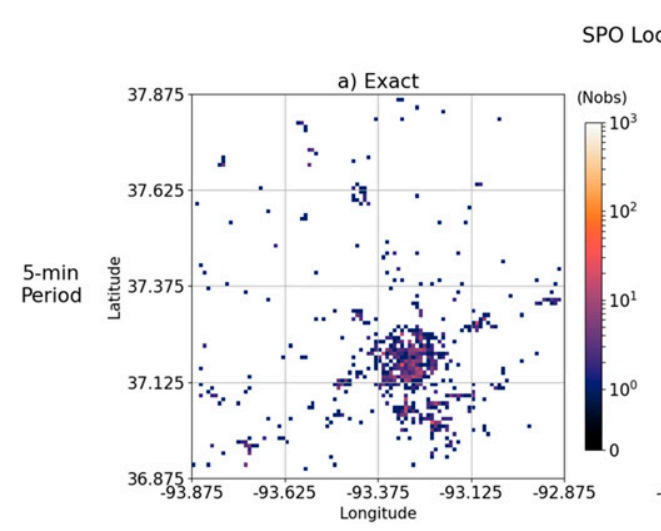

SPO Locations at Each Stage of Anonymization
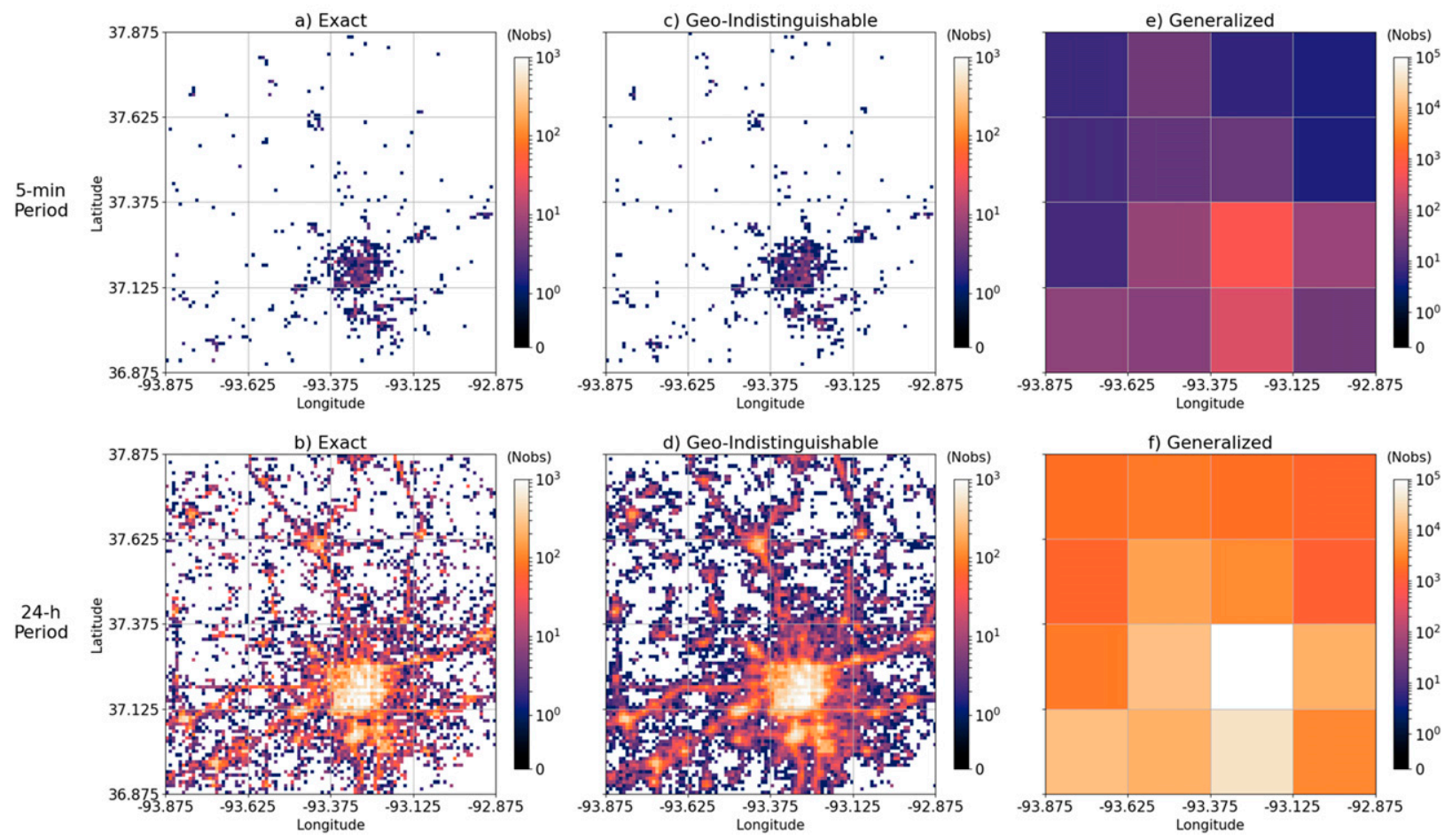

FIG. 3. 2D histograms of SPO locations at each stage of anonymization for a (top) 5-min period and (bottom) 24-h period. (a),(b) The distribution of exact (true) smartphone observation locations. (c),(d) The application of geo-indistinguishability. (e),(f) The spatiotemporal discretization (generalization) of geo-indistinguishable SPO locations that are rounded to the nearest $0.25^{\circ}$.

\section{a. MADIS pressure analyses}

To quantify smartphone surface pressure errors, a reference pressure analysis was generated using MADIS pressure observations. This analysis utilized multiresolution kriging (Nychka et al. 2015), a state-of-the-art method for the interpolation of large geospatial datasets. In multiresolution kriging, the pressure analysis is estimated by a series of compact, multiresolution basis functions and a Markov random field model for basis coefficients. This approach is advantageous for interpolating irregularly spaced observations since a multiscale covariance model can adjust to differences in station spacing.

The R package LatticeKrig (Nychka et al. 2016) was used to perform multiresolution analysis of MADIS pressure observations. Gridded terrain elevation was derived from the NOAA High-Resolution Rapid Refresh system (HRRR; Alexander et al. 2017; Blaylock et al. 2017), and two levels of basis functions were used to approximate large and smallscale covariances (Fig. 6a). The resolution of the kriging analysis was $0.05^{\circ} \times 0.05^{\circ}$. Since elevation data needed to be interpolated to the kriging grid, HRRR terrain elevation was chosen over higher resolution datasets like the USGS DEM (Gesch et al. 2014). The parameters of the LatticeKrig model used to generate pressure analyses are listed in Table 2. Crossvalidation experiments were performed with MADIS pressure observations to determine which parameter settings produced the most accurate analysis [lowest root-meansquared error (RMSE)].

MADIS pressure analyses, with a spatial resolution of $0.05^{\circ}$, were generated every 5 min between 15 August 2017 and 30 December 2018. The analysis domain covered most of the central and eastern United States $\left(28.5^{\circ}-48.5^{\circ} \mathrm{N}, 105.5^{\circ}-\right.$ $\left.70.5^{\circ} \mathrm{W}\right)$. Thirty conditional simulations were performed to estimate the uncertainty (i.e., prediction error) of pressure analyses generated with LatticeKrig. Specifically, the kriging standard error was computed by calculating the standard deviation of altimeter for the conditional simulations. This calculation was performed at each grid point producing a gridded analysis of prediction error (Fig. 6d).

An example MADIS pressure analysis is shown in Fig. 6c for the northeastern United States. The pressure analysis captured a local pressure minima northwest of Washington, D.C., as well as a pressure gradient across northern New Jersey and southern New York. Both features are visible in the 5-min point observations (Fig. 6b). The estimated kriging prediction error, displayed in Fig. 6d, demonstrates that the uncertainty of the analysis is highly dependent upon the local observation density, in agreement with prior literature using conventional variational data assimilation (Tyndall and Horel 2013).

Due to the different observing frequencies of METAR stations, the local observation density varies over time. Every $20 \mathrm{~min}$, the number of available METAR observations doubles (Fig. 1a). As a result, 5-min MADIS pressure analyses lack 
a) Minimum K-Anonymity

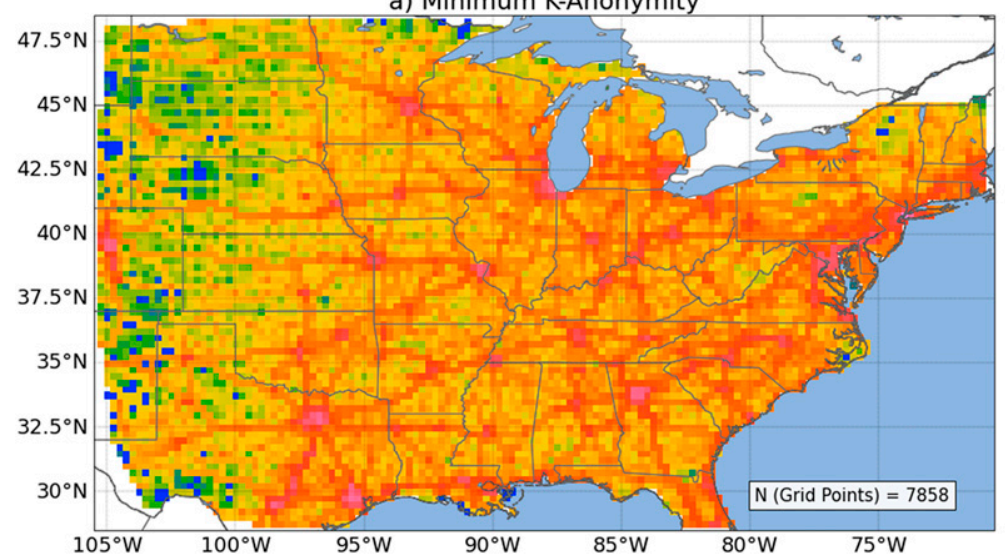

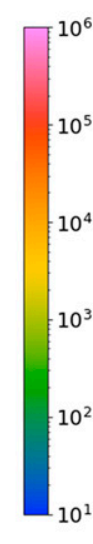

b) Distribution (May, 2018)

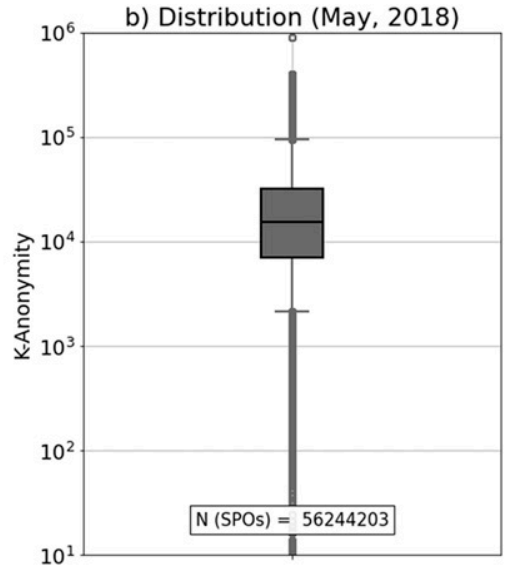

FIG. 4. (a) The minimum $k$-anonymity of SPOs at grid points spaced every $0.25^{\circ}$ valid for the full analysis period (15 Aug $2017-30$ Dec 2018). (b) The distribution of $k$-anonymity for 5-min SPOs anonymized during May 2018. The lower and upper whiskers of the boxplot depict the 5th and 95th percentiles, respectively. The black line bisecting the boxplot represents the median of the distribution.

temporal consistency as the spatial distribution of observations contributing to each analysis changes over time. To ameliorate this issue, a Rauch-Tung-Striebel (RTS) smoother was applied to the time series of MADIS pressure analyses at each grid point. In a real-time setting, a Kalman filter would be used in place of an RTS smoother. ${ }^{3}$ This technique for generating spatiotemporal pressure analyses, using multiresolution kriging and Kalman smoothing, is demonstrated in meteo-krig, an open-source GitHub repository (https://github.com/cmac994/meteo-krig).

\section{b. SPO error quantification}

SPO errors were estimated by binning SPOs into 5-min windows and bilinearly interpolating 5-min MADIS pressure analyses to the location of each SPO for that 5-min period. For SPOs with a UID, the precise latitude/longitude from the smartphone GPS is available. In contrast, anonymous ANON SPOs were binned into $0.25^{\circ}$ grid boxes during generalization. ANON-SPO errors were then estimated by interpolating MADIS pressure analyses to the central location of ANON grid boxes. Superobservations (superobs) were generated at these ANON grid points by averaging all ANON SPOs within each grid box. Aggregate statistics of anonymous SPOs sharing the same ANON grid point were computed during superobbing, including the mean, sum, standard deviation, skewness, and kurtosis of the altimeter setting and horizontal location accuracy. SPOs whose pressure exceeded three standard deviations from the mean were discarded to prevent superobs from being skewed by extreme outliers.

\section{c. Bias correction}

In McNicholas and Mass (2018a), random forests were trained to predict real-time SPO errors using sensor data from

\footnotetext{
${ }^{3}$ The process uncertainty was derived from the variance of altimeter setting analysis (i.e., kriging standard error). The observation uncertainty was set to $1 \mathrm{hPa}$. These settings were chosen by cross validating Kalman-smoothed MADIS analyses to determine which settings produced the analysis with the lowest RMSE.
}

the smartphone. In this study, the extreme gradient boosting (XGB; Chen and Guestrin 2016) machine learning model was used to predict SPO errors for the following month, from January to December 2018. During each month, smartphone pressure observations were bias corrected using SPO error predictions from XGB models trained on prior data. UID SPOs were trained using data from the previous eight months while ANON SPOs were trained using data from all prior months. Table 3 illustrates the training and testing periods during the first three months of 2018. Historical biases were calculated by subtracting observed pressures (from interpolated MADIS) from SPOs. Following McNicholas and Mass (2018a), only smartphones contributing at least 1000 SPOs during the training period were eligible for XGB bias correction. A list of variables used to train XGB models for ANON and UID SPOs is provided in Tables 4 and 5. One hundred trees were used in each XGB model, and the maximum tree depth was limited to three and the learning rate was set to 0.1. To bias correct UID SPOs, one XGB model was trained per UID (i.e., smartphone) to account for the unique behavior of individual smartphones. In contrast, ANON SPOs were biased-corrected by training an XGB model at each ANON grid point over which SPOs were anonymized.

Following McNicholas and Mass (2018b), SPOs from UID smartphones ineligible for bias correction with machine learning due to low observation counts were clustered by location using density-based spatial clustering of applications with noise (DBSCAN; Ester et al. 1996). During the training period, smartphones contributing between 100 and 1000 SPOs were clustered with DBSCAN to retrieve a list of frequented locations. A cluster was formed if at least 50 SPOs fell within a $60-\mathrm{m}$ neighborhood during the training period. The mean SPO pressure error was stored for each cluster location and subsequently used to predict pressure errors when, during the prediction period, an SPO fell within a DBSCAN cluster. Additional methods of bias correction were performed for comparison with the machine learning approach outlined above. These methods involved computing the average SPO error during training (AVG), using the most 


\section{SPO Bias Correction and Quality Control}

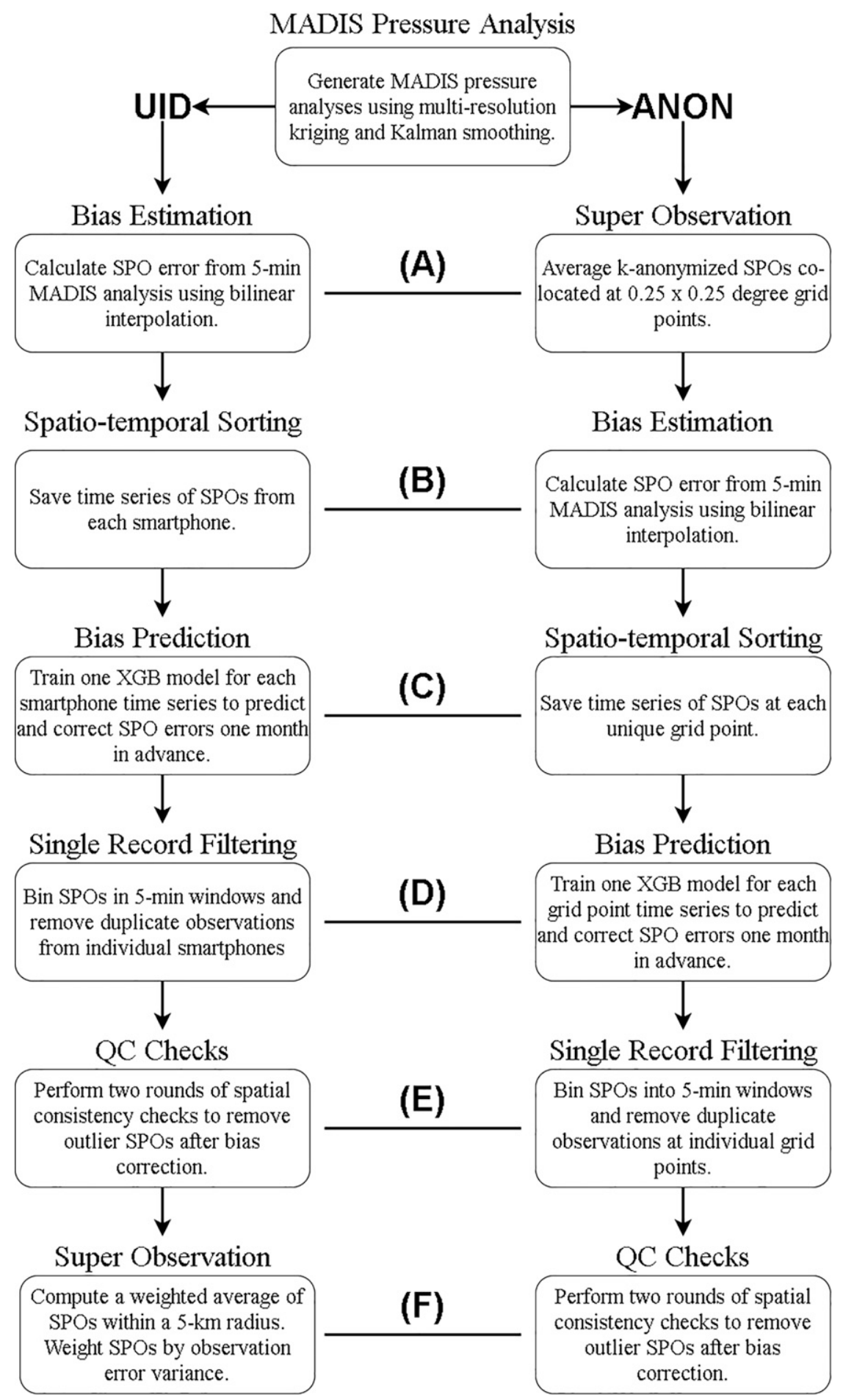

FIG. 5. The workflow of SPO data processing in this figure illustrates the procedure by which UID SPOs and ANON SPOs are bias corrected and quality controlled. 
Pressure Analysis: 2035 UTC 15 May 2018
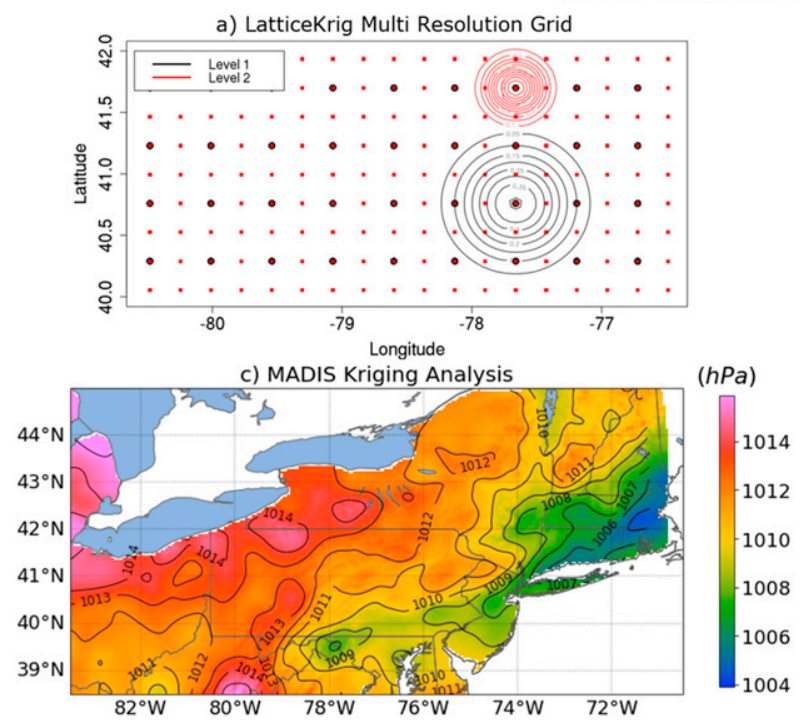

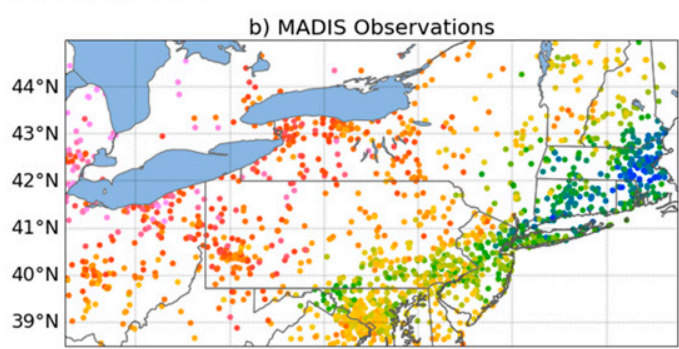

d) MADIS Kriging Standard Error

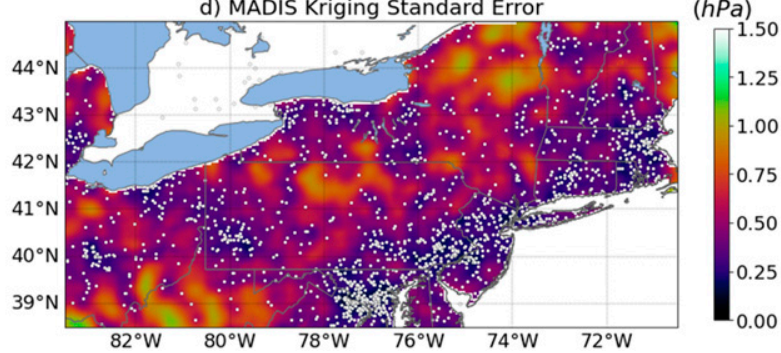

$(h P a)$

FIG. 6. (a) Two levels of LatticeKrig multiresolution basis functions on a latitude-longitude grid. (b) MADIS altimeter observations in the coastal mid-Atlantic states during the 5-min period centered at 2035 UTC15 May 2018. (c) MADIS kriging analysis of altimeter setting generated from MADIS pressure observations. (d) The prediction error of MADIS pressure analyses, estimated from 30 conditional simulations. White dots indicate the location of MADIS pressure observations contributing to the kriging analysis.

recent SPO error to predict the current SPO error (PERSIST), and using multivariate linear regression (LR) in place of an XGB model to predict SPO errors.

Together, the DBSCAN clustering approach and the XGB algorithm aim to provide the best-case scenario for bias correction of UID SPOs. While this approach is problematic due to privacy concerns (Boukoros et al. 2019) it is important to define the best-case scenario for SPO bias correction. Doing so allows for a comparison of bias-correction performance with ANON SPOs, facilitating a quantitative analysis of the degree to which SPO quality is reduced when SPOs are anonymized.

Bias correction of UID SPOs was performed on smartphone separately. Most smartphones only contributed observations over a portion of the collection period (August 2017-December 2018). On average, the lifetime of a smartphone in the IBM network was approximately 6 months (180 days). For this reason, the training period for UID SPOs was limited to a

TABLE 2. List of parameters used to initialize LatticeKrig multiresolution pressure analyses.

\begin{tabular}{|c|c|c|}
\hline Parameter & Description & Value \\
\hline $\mathrm{NC}$ & $\begin{array}{l}\text { Number of lattice points in first level and } \\
\text { along the largest dimension }\end{array}$ & 75 \\
\hline nlevels & $\begin{array}{l}\text { Number of levels for the } \\
\text { multiresolution basis }\end{array}$ & 2 \\
\hline a.wght & $\begin{array}{l}\text { The weight given to the central lattice } \\
\text { point in the spatial autoregression }\end{array}$ & 4.5 \\
\hline lambda & $\begin{array}{l}\text { Smoothing parameter (noise-to-signal } \\
\text { ratio) }\end{array}$ & 0.5 \\
\hline alpha & $\begin{array}{l}\text { Relative variances for the multiresolution } \\
\text { levels. }\end{array}$ & $0.1,0.9$ \\
\hline
\end{tabular}

maximum of 8 months. Extending the maximum training period beyond 8 months increased computation time without improving bias-correction performance. ANON SPOs were bias corrected by location (grid point). Although the number of UID SPOs declined later in the study period, the number of ANON SPOs remained consistent since they were produced through superobbing. The training period for ANON SPOs incorporated data from all prior months. This ensured that the bias correction of ANON SPOs could account for the fact that smartphones contributing to ANON SPOs changed over time (as smartphones left and entered the network).

\section{d. Quality control}

QC measures were applied to both ANON and UID SPOs after bias correction to remove outliers due to erroneous XGB model predictions. Retrieved within a 5-min time window, collocated SPOs were filtered by retaining only the SPO closest in time to the center of the time window. QC checks were applied to the remaining SPOs, beginning with a validity check to remove prominent outliers. Specifically, SPOs with an altimeter setting falling outside the 890-1060-hPa range were flagged and removed. Next, a gross spatial check (Madaus and Mass 2017) and a spatial consistency check (Miller and Benjamin 1992) were applied to remove SPOs in substantial disagreement with surrounding observations. Specifically, nearby SPOs were divided into four quadrants. Up to six SPOs were retrieved from each quadrant and distance-weighted interpolated to the location of the SPO being checked. The standard deviation of altimeter from the surrounding SPOs was also computed. If the difference between the SPO and the interpolated observation was greater than twice this standard deviation, then the SPO was rejected as an outlier. 
TABLE 3. The breakdown of training $(\mathrm{T})$ and testing $(\mathrm{P})$ periods each month. XGB models are retrained every month to extend the training period and predict SPO errors for the next month. For UID SPOs, training periods were limited to a maximum of 8 months, before the start of the testing period.

\begin{tabular}{ccccccccccc}
\hline \hline \multicolumn{2}{c}{ Month and year } & Aug 2017 & Sep 2017 & Oct 2017 & Nov 2017 & Dec 2017 & Jan 2018 & Feb 2018 & Mar 2018 & Apr 2018 \\
\hline Prediction month & Jan & T & T & T & T & T & P & & & \\
& Feb & T & T & T & T & T & T & P & \\
& Mar & T & T & T & T & T & T & T & P & T \\
& Apr & T & T & T & T & T & T & T & P \\
\hline
\end{tabular}

\section{e. Smartphone pressure analyses and superobs}

After bias correction and QC, both UID and ANON SPOs were used to generate separate smartphone pressure analyses. These analyses were generated using the same multiresolution kriging and Kalman-smoothing technique employed to create MADIS pressure analyses. Smartphone pressure analyses were generated every 5 min between 1 January 2018 and 30 December 2018. These analyses were produced for the same domain as MADIS pressure analyses.

Nearly half of UID SPOs were spaced closer than the $\sim 5$-km resolution of multiresolution kriging analyses (Fig. 1b). Consequently, UID smartphone pressure analyses were generated with superobs. These superobs were produced by calculating a weighted average of UID SPOs within a $5-\mathrm{km}$ radius, with the weighting based on the SPO's observation error variance, estimated using the root-mean-square error of XGB model predictions and the MADIS kriging prediction errors at the location of the SPO. Weighting UID SPOs by observation error variance allowed superobs to reflect the uncertainty of bias estimation and correction. ANON SPOs were simply averaged as they were superobbed before bias estimation and correction (Fig. 5).

\section{Results}

\section{a. Performance of SPO bias correction and $Q C$}

Various bias-correction approaches were evaluated by calculating the RMSE of SPOs from each UID smartphone and at each ANON grid point. The performance of the various biascorrection methodologies is illustrated in Fig. 7, which displays the bias prediction error and observation count during training for all (UID) smartphones and ANON grid points. Since bias correction was performed monthly (Table 3), the median altimeter RMSE was computed each month. For UID smartphones contributing between 100 and 1000 observations during training, bias correction with DBSCAN clustering reduced the median altimeter RMSE by approximately $23 \%$, relative to uncorrected SPOs. For these smartphones, a decrease in the median altimeter RMSE was coincident with an increase in the median observation count during the spring and summer months (Fig. 7). During these months, SPOs were more likely to be retrieved at ground level as people spend more time outside (Leech et al. 2002).

Figure 7c shows the median altimeter RMSE for the UID smartphones that contributed more than 1000 observations during training. In contrast to Fig. 7a, error statistics for these smartphones were more consistent throughout the year, in part due to the considerably larger number of observations per smartphone (Fig. 7d). In general, all bias-correction methodologies reduced smartphone (UID) bias prediction error (Fig. 7c). The machine learning approach (XGB) outperformed all other methods, producing a $57 \%$ reduction in the median smartphone altimeter RMSE. Other methods of bias correction such as PERSIST, AVG, and multivariate LR decreased altimeter RMSE by $29 \%, 41 \%$, and $46 \%$, respectively. In the final months of 2018, the XGB and LR median altimeter RMSE increased as observation counts declined. The number of observations, available during training, reached a peak in April 2018 before steadily declining over the remainder of the year.

In contrast to UID smartphones, the average number of pressure observations available during training increased monotonically over time at ANON grid points (Fig. 7f). Counterintuitively, this did not result in a reduction in bias-corrected SPO error. While the number of SPOs available during training steadily increased, specific smartphones joined and left the pressure network over time. As a result, the bias correction of ANON SPOs showed little improvement as the length of the training period increased. Nevertheless, all bias-correction methodologies reduced the altimeter RMSE of ANON SPOs, except for persistence. This result is not surprising since superobbed SPOs at ANON grid points are frequently drawn from a changing set of smartphones. Since ANON SPOs were superobbed before bias correction, their uncorrected altimeter RMSE (Fig. 7e) was typically lower than for individual UID smartphones (Fig. 7c). Consequently, the bias correction of ANON SPOs resulted in smaller reductions in altimeter RMSE. Bias correction through simple averaging reduced median altimeter RMSE by $12 \%$ while linear regression and XGB machine learning decreased altimeter RMSE by $20 \%$ and $43 \%$, respectively. For ANON SPOs, the difference in bias-corrected altimeter RMSE between XGB and LR was double that observed during the bias correction of UID smartphones. This suggests that the machine learning approach to bias correction has greater efficacy, relative to conventional methods when applied to aggregate SPOs from more than one smartphone.

In Fig. 8, SPO errors were averaged spatially across the analysis domain. The domain average RMSE of SPOs was estimated using MADIS pressure analyses. The RMSE of SPOs was computed every $5 \mathrm{~min}$, from which the median altimeter RMSE for each month was retrieved. Figure 8a displays the median altimeter RMSE for all UID SPOs, before 
TABLE 4. Description of UID SPO variables used to train XGB models.

\begin{tabular}{ll}
\hline \multicolumn{1}{c}{ Variables } & \multicolumn{1}{c}{ Description } \\
\hline time & Time since start of UNIX epoch \\
loc_accuracy & Horizontal location accuracy \\
elev & Terrain elevation from USGS DEM \\
elev_std & Two standard deviations of the nine nearest \\
& USGS DEM grids \\
pressure & Surface pressure \\
altimeter & Sea level pressure \\
Hour & Hour of day (0-24) \\
Weekday & Day of week (0-7) \\
Day of year & Number of days since 1 Jan (0-365) \\
time_dt & Time (s) since last observation \\
horizontal_ & Change in horizontal location accuracy \\
accuracy_dt & between current and previous observation \\
elev_dt & Elevation difference between current and \\
& previous observation \\
elev_std_dt & Difference in elevation standard deviation \\
& since the last observation \\
pressure_dt & Pressure change since last observation \\
altimeter_dt & Altimeter change since last observation \\
\hline
\end{tabular}

bias correction (uncorrected), after bias correction, and after QC. For UID SPOs, the domain-averaged altimeter RMSE was higher than the median smartphone altimeter RMSE (Fig. 8). This occurred because, in the domain average, altimeter RMSE was positively skewed by infrequent outliers that deviated substantially $(>10 \mathrm{hPa}$ ) from the MADIS pressure analysis. These outliers were not always suppressed during bias correction since XGB does not guarantee a reduction of SPO error. On average, for any given UID smartphone, XGB bias correction reduced SPO errors approximately $87 \%$ of the time.

Both Figs. $8 \mathrm{a}$ and $8 \mathrm{c}$ show a remarkable reduction in the domainaveraged altimeter RMSE, for both UID and ANON SPOs, after bias correction and quality control. Relative to uncorrected SPOs, the monthly median altimeter RMSE across the analysis domain was reduced by $85 \%$ and $43 \%$ for UID and ANON SPOs, respectively. This reduction in SPO error was achieved without substantially reducing observation counts. Approximately $33 \%$ of bias-corrected UID SPOs (Fig. 8b) and 3\% of bias-corrected ANON SPOs (Fig. 8d) were removed after failing spatial-consistency QC checks. As in Fig. 8, the reduction in observation count and domain-averaged altimeter RMSE was smaller for ANON SPOs than for UID SPOs because ANON SPOs were superobbed before XGB bias correction. Superobbing reduces uncertainty and constrains outliers, reducing the error of uncorrected SPOs.

\section{b. Smartphone pressure analysis: Spatiotemporal verification}

After bias correction and quality control, 5-min pressure analyses were produced using UID and ANON SPOs, as outlined in section 3. Observed pressures at METAR sites were used for verification. Verification sites were spatially filtered to ensure that no selected sites were spaced closer than $25 \mathrm{~km}$. To compare smartphone pressure analyses to MADIS analyses,
TABLE 5. Description of ANON SPO variables used to train XGB models. Bulk statistics (e.g., mean, skewness) were calculated from anonymous SPOs assigned the same grid point during generalization.

\begin{tabular}{|c|c|}
\hline Variables & Description \\
\hline time_grid & $\begin{array}{l}\text { Time since start of UNIX epoch (rounded } \\
\text { to the nearest fifth minute) }\end{array}$ \\
\hline loc_accuracy_mean & Mean horizontal location accuracy \\
\hline loc_accuracy_std & $\begin{array}{l}\text { Standard deviation of horizontal location } \\
\text { accuracy }\end{array}$ \\
\hline alts_sum & Sum of altimeter setting \\
\hline alts_mean & Mean altimeter setting \\
\hline alts_std & Standard deviation of altimeter setting \\
\hline alts_skew & Skewness of altimeter setting \\
\hline alts_kurt & Kurtosis of altimeter setting \\
\hline time_grid_dt & $\begin{array}{l}\text { Time (s) since last observation (rounded } \\
\text { to the nearest } 5 \text { th minute) }\end{array}$ \\
\hline $\begin{array}{l}\text { horizontal_accuracy_ } \\
\text { mean_dt }\end{array}$ & $\begin{array}{l}\text { Change in mean horizontal location } \\
\text { accuracy between current and previous } \\
\text { observation }\end{array}$ \\
\hline $\begin{array}{l}\text { horizontal_accuracy_ } \\
\text { std_dt }\end{array}$ & $\begin{array}{l}\text { Change in standard deviation of } \\
\text { horizontal accuracy between current } \\
\text { and previous observation }\end{array}$ \\
\hline alts_sum_dt & $\begin{array}{l}\text { Change in altimeter sum since the last } \\
\text { observation }\end{array}$ \\
\hline alts_mean_dt & $\begin{array}{l}\text { Change in mean altimeter since the last } \\
\text { observation }\end{array}$ \\
\hline alts_std_dt & $\begin{array}{l}\text { Change in standard deviation of altimeter } \\
\text { since the last observation }\end{array}$ \\
\hline alts_skew_dt & $\begin{array}{l}\text { Change in skewness of altimeter setting } \\
\text { since the last observation }\end{array}$ \\
\hline alts_kurt_dt & $\begin{array}{l}\text { Change in kurtosis of altimeter setting } \\
\text { since the last observation }\end{array}$ \\
\hline Hour & Hour of day (0-24) \\
\hline Weekday & Day of week $(0-7)$ \\
\hline Day of year & Number of days since 1 Jan (0-365) \\
\hline
\end{tabular}

fourfold cross validation was employed. Four MADIS-based pressure analyses were generated, with a quarter of MADIS observations withheld in each analysis. For each analysis, error statistics were calculated at withheld METAR verification sites. In addition to MADIS analyses, altimeter analysis from the HRRR (Blaylock et al. 2017) was also retrieved for comparison with smartphone pressure analyses.

To evaluate the performance of pressure analyses over time, the monthly median domain-averaged altimeter RMSE was calculated for the MADIS and smartphone pressure analyses (Fig. 9a). Error statistics were produced for smartphone pressure analyses generated using ANON SPOs (SMART-ANON analyses), 5-km superobbed UID SPOs before bias correction (SMART-BIAS analyses), and 5-km superobbed UID SPOs after bias correction/QC (SMART-UID analyses). Even without bias correction, superobbing UID SPOs produced smartphone pressure analyses with a median annual altimeter RMSE of approximately $1 \mathrm{hPa}$ (Fig. 9b). Although the SMART-BIAS analyses underperformed relative to MADIS analyses, the range of SMART-BIAS domain-averaged altimeter RMSE fell between 0.9 and $1.35 \mathrm{hPa} 90 \%$ of the time (Fig. 9b). This suggests that superobbing SPOs, without bias correction, could 
Bias Prediction Error (Testing) and Observation Counts (Training)
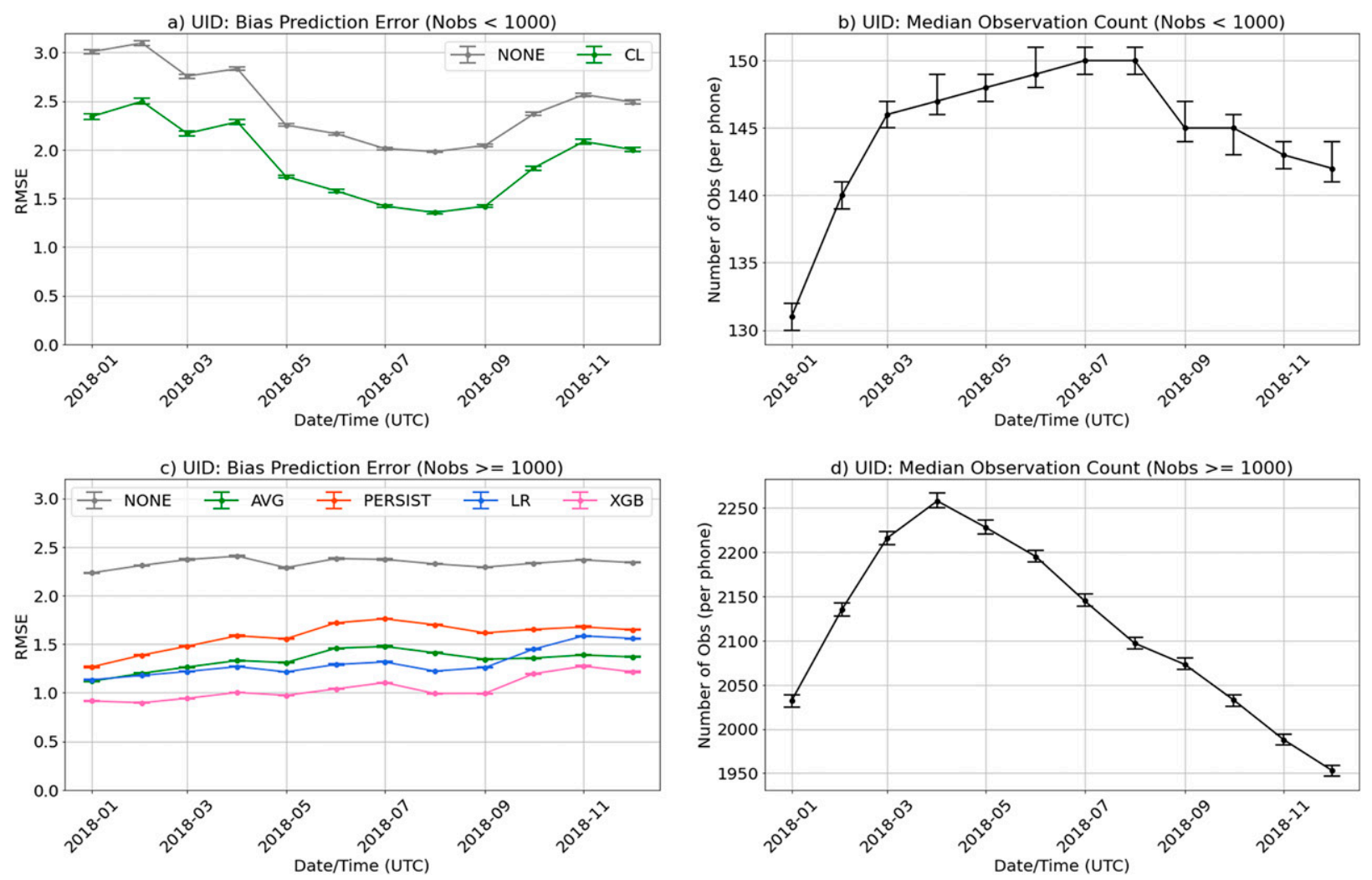

e) ANON: Bias Prediction Error
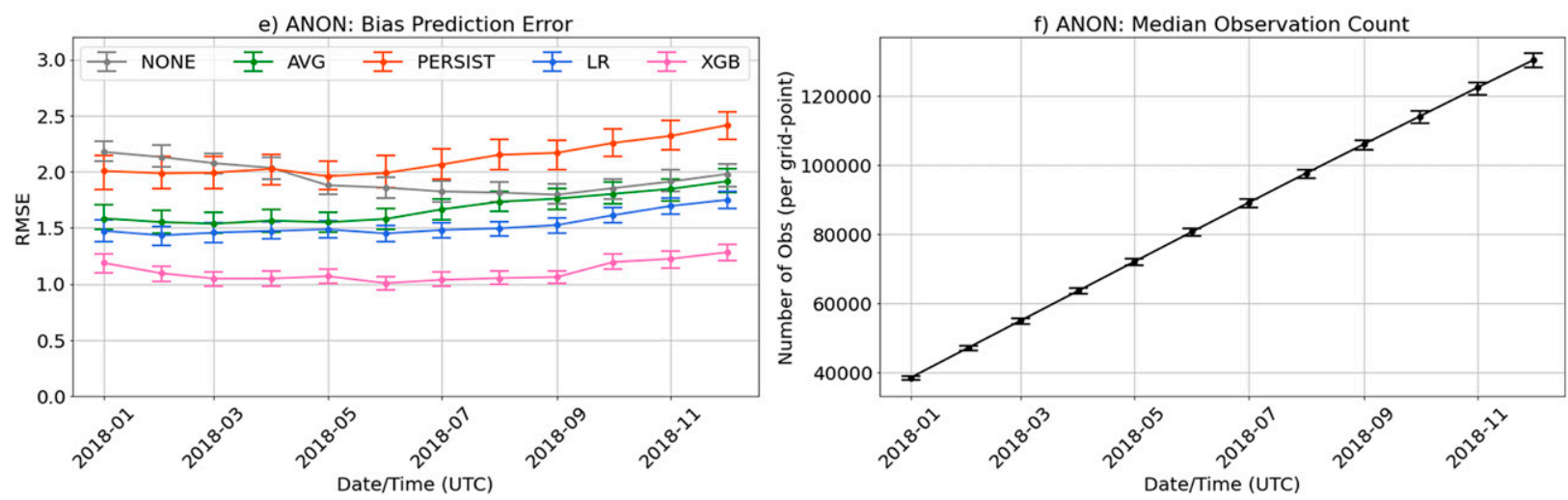

FIG. 7. Smartphone (left) bias prediction error and (right) median observation counts, during testing and training periods, respectively. DBSCAN clustering (CL) was used to predict SPO pressure errors (a) for UID smartphones contributing fewer than 1000 observations during training. Error statistics for (c) UID and (e) ANON smartphones. SPO errors were predicted using XGB, multivariate LR, AVG, and using PERSIST. Observation counts for (d) UID and (f) ANON SPOs show the median number of observations retrieved during training, for each smartphone, and at each anonymous grid point, respectively. Vertical bars depict $95 \%$ bootstrapped confidence intervals for the median observation count and bias prediction RMSE.

potentially provide useful pressure data in regions where in situ observations are especially sparse.

Throughout the year-long analysis period, SMART-UID and SMART-ANON analyses consistently outperformed MADIS analyses. In the annual average, the domain-averaged altimeter RMSE of SMART-ANON analysis was reduced by $21 \%$ relative to MADIS analysis. In contrast, SMART-UID analyses reduced domain-averaged altimeter RMSE by $32 \%$, relative to MADIS analyses (Fig. 9b). This difference in performance between smartphone pressure analyses produced with ANON SPOs and UID SPOs is only $34 \%$, implying that the anonymization of SPOs only modestly degrades their quality and efficacy.

Monthly differences in SMART-ANON and SMART-UID analysis error are shown in Fig. 9a. In July, the domain-averaged 
Domain-averaged Bias Prediction Error and Observation Count
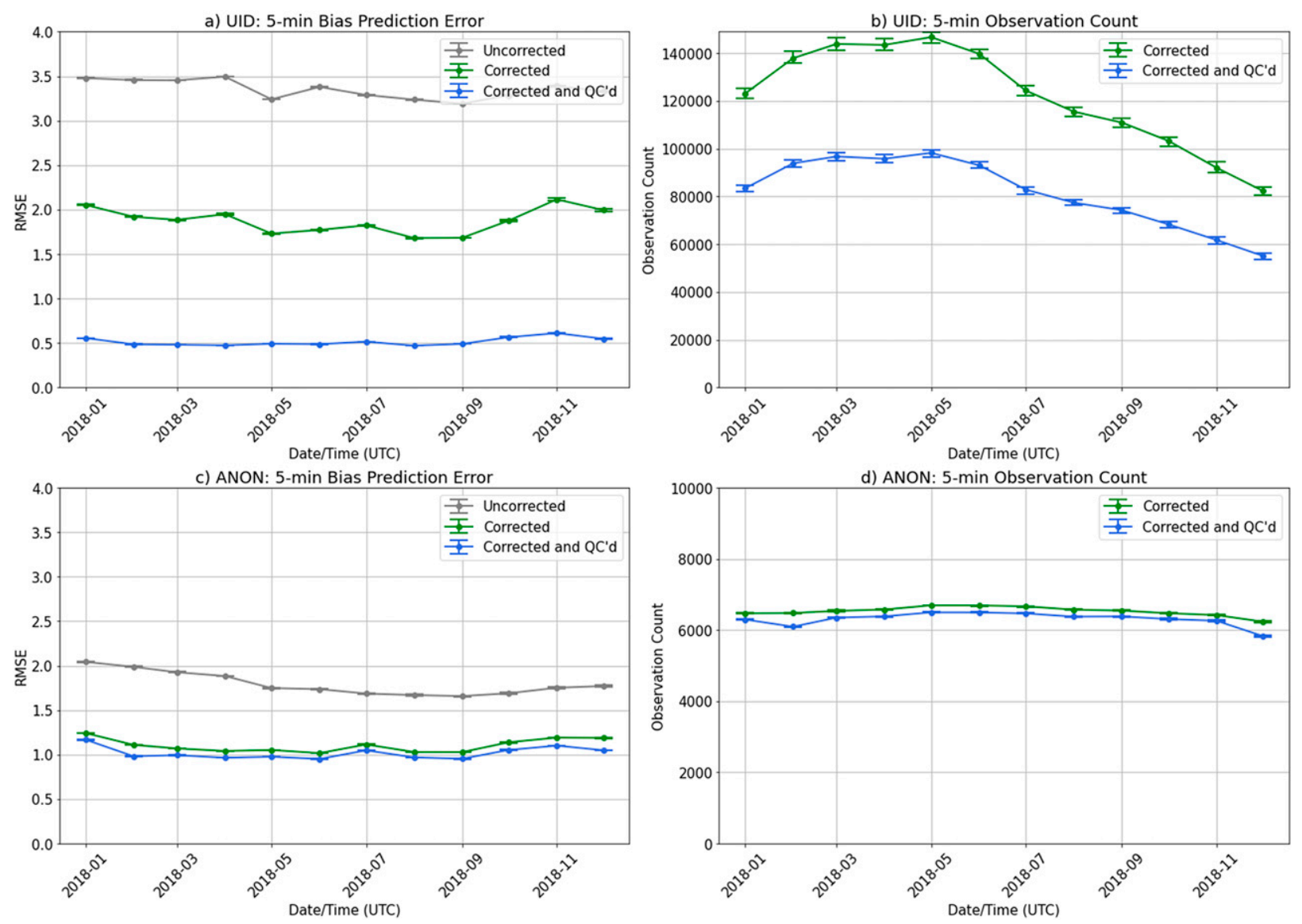

FIG. 8. Domain-averaged bias prediction error and observation count for (top) UID and (bottom) ANON SPOs. The RMSE, for the analysis domain was computed using SPOs retrieved during 5-min observation windows. The median monthly RMSE, is displayed for (a) UID and (c) ANON SPOs before bias correction (gray), after bias correction (green), and after additional QC checks (blue). The median 5-min observation count is shown for (b) UID and (d) ANON SPOs, before and after QC checks. Vertical bars depict 95\% bootstrapped confidence intervals for the monthly median observation count and bias prediction RMSE.

altimeter RMSE increased for both SMART-ANON and SMART-UID analysis and subsequently remained relatively high. This increase in analysis error, which was also observed in SPO error statistics from Fig. 8, may be attributed to changes in measurement signal-to-noise ratio and declining observation counts. In the summer, synoptic pressure variations reach a minimum amplitude and mesoscale pressure variances become more pronounced (Jacques et al. 2015). This change in signal-to-noise ratio complicates machine learning predictions of SPO error. Since only a single year of data was available, SPO bias correction in July was performed using XGB models trained on data retrieved during the preceding fall, winter, and spring when synoptic pressure variations were larger and synoptic-scale features more dominant. In August 2018, domain-averaged altimeter RMSE error declined as the training period extended into the summer, incorporating SPO errors from periods when mesoscale pressure variation was more pronounced. During the fall of 2018, the domain-averaged altimeter RMSE for SMARTANON and SMART-UID analysis remained elevated, relative to the previous winter and spring, as the number of observations of bias-corrected and QC'd SPOs steadily declined.

Figure 10a highlights the impact of superob spacing on analysis altimeter RMSE. In this figure, MADIS, SMART-BIAS, and SMART-UID pressure analyses were reproduced using superobs with increasing station spacing. Results for ANON SPOs, which were superobbed at a resolution of $25 \mathrm{~km}$ before bias correction, are provided for comparison. SMART-BIAS altimeter RMSE steadily increased with increasing superob spacing as the lack of QC increased the likelihood of extreme outliers degrading superob quality. The performance of SMARTUID and MADIS analysis was relatively unchanged at superob spacings below 25 and $50 \mathrm{~km}$, respectively. This was a consequence of the resolution of the verification (METAR) observations, which were spatially filtered to a minimum station spacing of $25 \mathrm{~km}$ to ensure geographic diversity. At a superob spacing of $25 \mathrm{~km}$, altimeter RMSE was $1.20 \mathrm{hPa}$ for SMART-BIAS analysis, $0.95 \mathrm{hPa}$ for MADIS analyses, $0.76 \mathrm{hPa}$ for SMART-ANON analysis, and $0.67 \mathrm{hPa}$ for SMART-UID analysis (Fig. 10a). Interestingly, the average number of superobs contributing to 

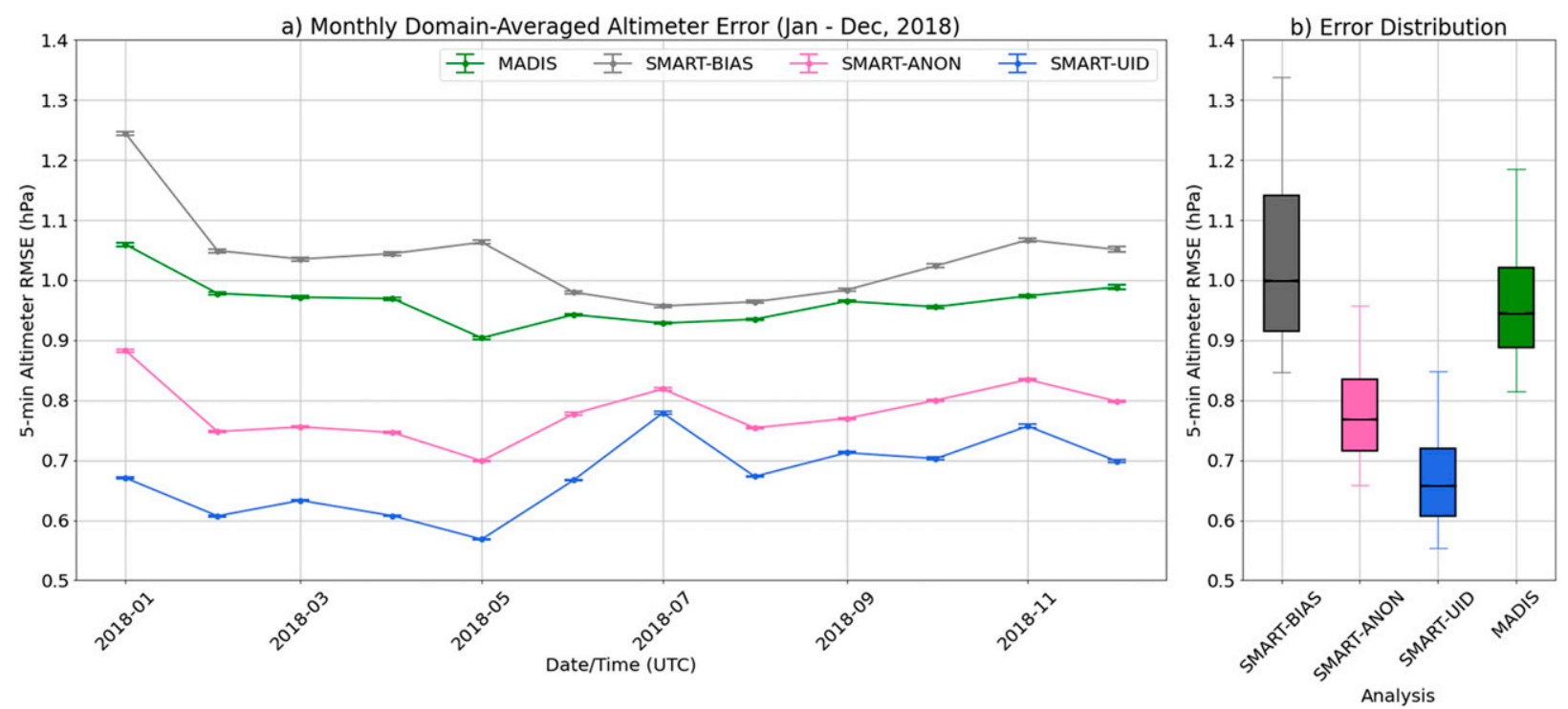

FIG. 9. Median domain-average altimeter error for kriging pressure analyses. (a) Monthly median domain-averaged altimeter RMSE, during 2018, for MADIS (green), and smartphone pressure analyses produced using uncorrected UID SPOs (gray), corrected UID SPOs (blue), and corrected ANON SPOs (pink). Vertical bars depict 95\% bootstrapped confidence intervals for the monthly median domainaveraged altimeter RMSE. (b) Boxplots depict the distribution of the domain-averaged altimeter RMSE, for each gridded pressure analysis, over the year-long analysis period. The lower and upper whiskers of each boxplot depict the 5th and 95th percentiles, respectively. The black line bisecting each boxplot represents the median of the distribution.

pressure analysis was greatest for SMART-ANON analyses (Fig. 10b). In contrast to SMART-UID and SMART-BIAS, which relied on SPOs with unique id2entifiers, SMART-ANON analyses were generated using all available SPOs, including those without unique identifiers.

To examine how pressure analysis error varied spatially, the annual altimeter RMSE was calculated at each METAR verification site. The difference in altimeter error between MADIS and smartphone pressure analyses was computed at all verification sites and displayed in Fig. 10. In this figure, verification stations were grouped by observation frequency with ASOS (circles) and AWOS (triangles) stations contributing observations every 5 and $20 \mathrm{~min}$, respectively. Compared to MADIS analyses, SMART-BIAS analyses exhibited larger altimeter error at most verification sites (Fig. 11a). Conversely, SMART-ANON and SMART-UID analyses showed an overall reduction in altimeter error, relative to MADIS.

At AWOS sites, the difference in altimeter error between SMART-ANON and MADIS analyses was negligible. Every $20 \mathrm{~min}$, when AWOS observations were available, the number of METAR observations contributing to MADIS analyses doubled, reducing MADIS altimeter error and eliminating the difference in error between SMART-ANON and MADIS analyses at AWOS sites (Fig. 11). A similar result was noted when SMARTUID analyses were compared to MADIS analyses. In the case of SMART-UID analyses, altimeter error was reduced relative to MADIS at a plurality of verification sites; however, the reduction in altimeter error was greatest at ASOS sites, where verification observations were available every 5 min (Fig. 11).

The difference in altimeter error at ASOS and AWOS sites highlights the superior temporal resolution of SPOs. A significant fraction of MADIS observations, especially those in rural areas, were not available at a high temporal frequency (every $5 \mathrm{~min}$ ). Due to superobbing, the number of ANON and UID SPOs contributing to SMART analysis changed little between 5-min analysis periods. Since the spatial and temporal resolution of the SPO network was more consistent than the MADIS network, SMART-UID (ANON) analyses outperformed MADIS analyses when low-frequency (AWOS) observations were unavailable. This increase in performance was only observed at ASOS sites since they provided verification data at times when AWOS data were unavailable.

In all analysis comparisons (Fig. 11), the greatest differences in altimeter RMSE were observed in regions where MADIS observations were sparser, such as in the upper Great Plains. In this region, the mean difference in altimeter RMSE between SMART-UID (ANON) and MADIS analysis was -0.43 $(-0.17) \mathrm{hPa}$. In contrast, where MADIS observation density was high and MADIS analyses well constrained, SMART analyses provided little additional benefit. In the northeastern United States, the mean difference in altimeter RMSE from MADIS was negligible for SMART-ANON analyses and around $-0.07 \mathrm{hPa}$ for SMART-UID analyses.

\section{c. Smartphone pressure analysis: Case studies}

As shown above, smartphone pressure analyses improved upon MADIS analyses in both spatially (Fig. 9) and temporally (Fig. 11) averaged error statistics. To evaluate how these improved analyses were expressed for severe weather events, two cases were examined. The first focused on the landfall of Hurricane Michael along the coast of the Florida Panhandle. An analysis of this event, near the time of landfall (1730 UTC 
Annual Domain-Averaged Altimeter Error and Ob Counts (Jan - Dec, 2018)
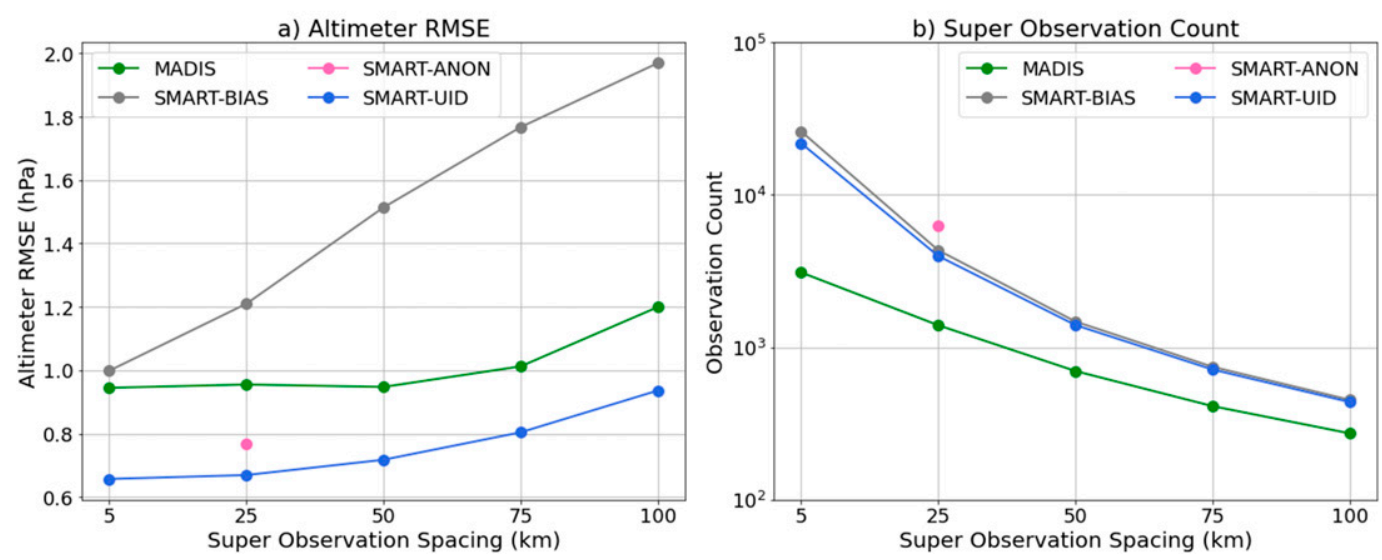

FIG. 10. Sensitivity of median domain-average altimeter error to super observation spacing. Median domainaveraged altimeter (a) RMSE and (b) observation count, during 2018, for MADIS (green), and smartphone pressure analyses produced using uncorrected UID SPOs (gray) and corrected UID SPOs (blue), superobbed at 5-, 25-, 50-, 75-, and 100-km resolution. Results for ANON SPOs, which were superobbed at a resolution of $25 \mathrm{~km}$ before bias correction, are displayed for comparison.

10 October 2018), is displayed in Fig. 12. Around this time, Michael's central pressure reached a minimum of $919 \mathrm{hPa}$ (Wadler et al. 2021). Although none of the sea level pressure analyses captured the full depth of Michael's low pressure center, SMART-UID and SMART-ANON analyses came closest to reproducing the observed pressure trace at Tyndall Air Force Base (AFB; Fig. 12). While individual SPOs recorded pressure measurements as low as $925 \mathrm{hPa}$, kriging and superobbing produced a minimum pressure of approximately $940 \mathrm{hPa}$ in SMART-ANON and SMART-UID analyses. The minimum observed pressure was slightly lower in SMARTUID analyses $(935 \mathrm{hPa})$ than SMART-ANON analyses $(942$ $\mathrm{hPa}$ ) since UID SPOs were superobbed at $5 \mathrm{~km}$, whereas ANON SPOs were superobbed before bias correction at a resolution of $\sim 25 \mathrm{~km}$. In the HRRR, MADIS, and SMARTBIAS analyses the minimum pressure remained above $960 \mathrm{hPa}$. Although KPAM (Tyndall AFB) was included in the MADIS pressure network, MADIS stopped receiving data from that site after 1600 UTC. This data loss may have contributed to the failure of HRRR and MADIS analyses to fully capture the depth of Michael's low pressure center. KPAM data, up to 1725 UTC (Fig. 12), were retrieved retroactively through MesoWest (Horel et al. 2002). The dearth of in situ station data during a high-impact weather event such as Hurricane Michael highlights the benefits of an extensive network of SPOs. Even as Michael made landfall and moved inland, pressure observations from smartphones declined but remained abundant.

A second case study (Fig. 13) presents pressure analyses and time series during the passage of a mesoscale convective system (MCS) over the upper Midwest on 18 September 2018. During the event, a 4-hPa increase in altimeter setting was observed at KYJG, an AWOS verification site in southern Minnesota (Fig. 12). While both SMART-BIAS and MADIS analyses captured the pressure signal of the MCS, they failed to resolve the tight pressure gradient along the leading edge of the storm and underestimated the amplitude of the mesohigh. Compared to MADIS analyses, both SMART-ANON and SMARTUID analyses better captured the amplitude of the pressure perturbation associated with the MCS. At 1300 UTC, there was remarkable consistency between SMART-ANON and SMART-UID pressure analyses, especially regarding the structure of the mesohigh. The relatively uniform distribution of ANON SPOs and the abundance of UID SPOs in the vicinity of the MCS explain the consistency between the two analyses (Fig. 12). Relative to SMART-UID analyses, the passage of the mesohigh was slightly delayed in SMARTANON analyses relative to the observed pressure trace at KYJG (Fig. 11g). This was a consequence of the coarser spatial resolution of ANON SPOs.

\section{Summary and conclusions}

This paper describes the bias correction, quality control, and the anonymization of smartphone pressure observations (SPOs) for application in both weather forecasting and research. This work takes advantage of over 30 billion SPOs that were retrieved from the Weather Channel (IBM) smartphone app. SPOs were anonymized through the spatial obfuscation (geoindistinguishability) and generalization ( $k$-anonymity) of location data. To evaluate SPO error, multiresolution kriging was employed to generate high-frequency ( $5 \mathrm{~min}$ ) MADIS sea level pressure analyses. Bias correction of both anonymous (ANON) and uniquely identified (UID) SPOs was performed using unsupervised clustering (DBSCAN; Ester et al. 1996), machine learning (XGB; Chen and Guestrin 2016), and conventional approaches such as persistence, averaging, and multivariate linear regression. The machine learning approach to bias correction outperformed all other methods for both ANON and UID SPOs, reducing the median smartphone pressure errors by 
a)

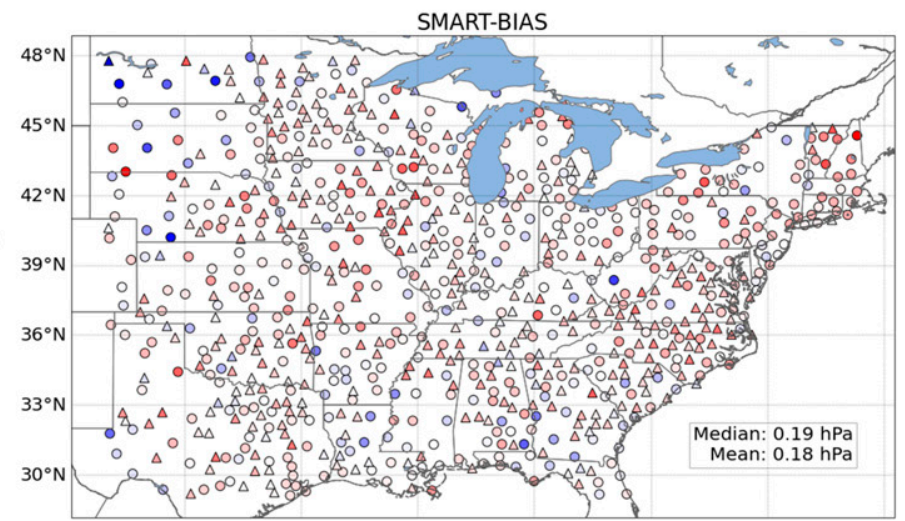

b)
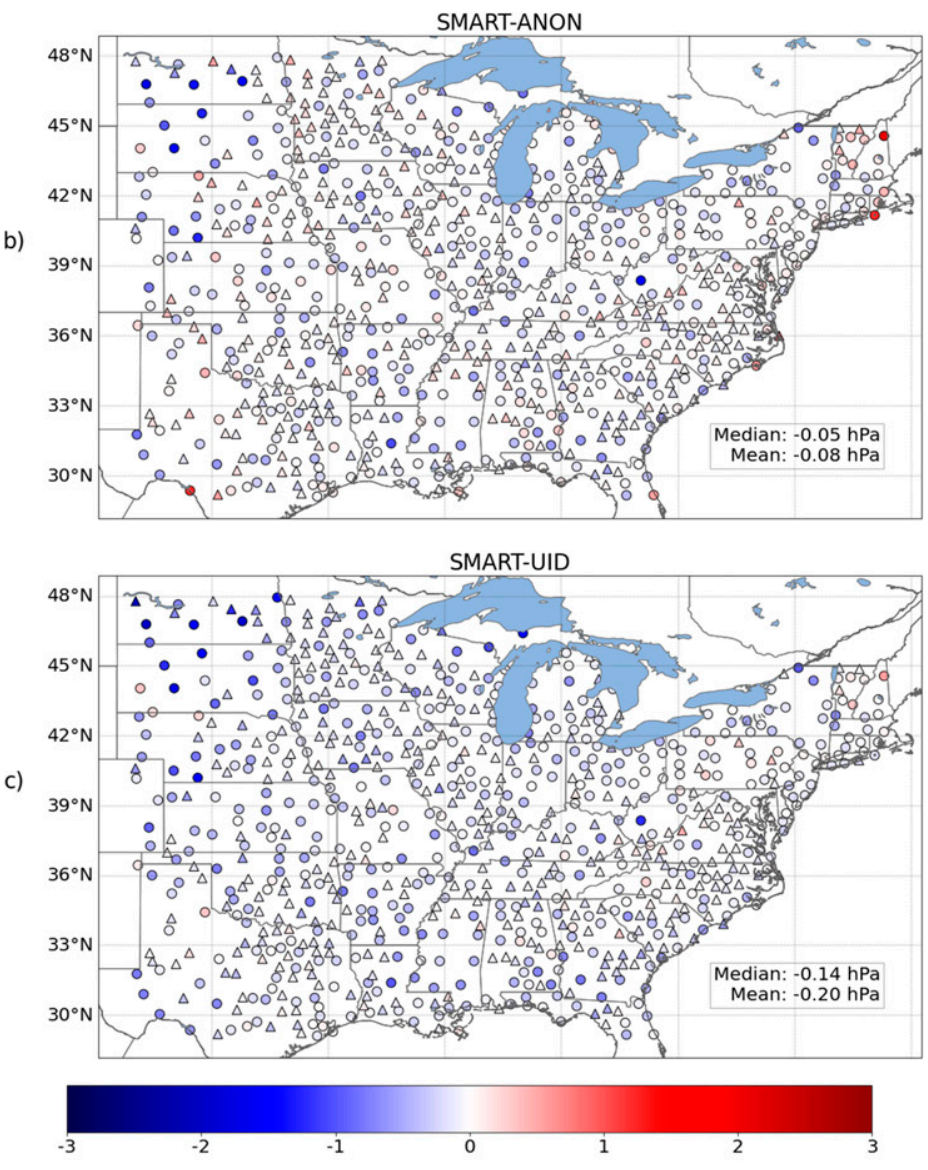
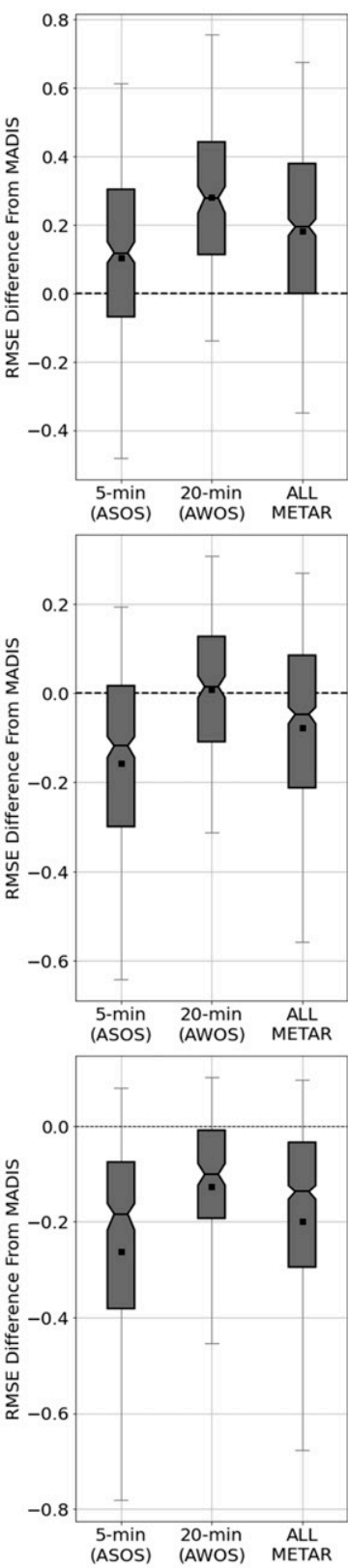

RMSE Difference from MADIS

FIG. 11. Difference in annual average altimeter RMSE between smartphone pressure analyses and MADIS pressure analyses. Smartphone pressure analyses were produced with uncorrected UID SPOs (SMART-BIAS), corrected UID SPOs (SMART-UID), and corrected ANON SPOs (SMART-ANON). The difference in annual average altimeter RMSE was computed by subtracting the MADIS analysis RMSE from smartphone analysis RMSE analyses. This altimeter RMSE difference is shown for (a) SMART-BIAS, (b) SMART-ANON, and (c) SMART-UID analyses. High-frequency $(5 \mathrm{~min})$ METARs from ASOS were marked as circles, while lowfrequency (20 min) METARs from AWOS were marked as triangles in scatterplots (a)-(c). On the right, the distribution of altimeter RMSE difference at all verification sites is shown by station type. The lower and upper whiskers of each boxplot depict the 5th and 95th percentiles, respectively. The black line bisecting each boxplot represents the median of the distribution. Notches on each boxplot show the $95 \%$ confidence interval for the median. 

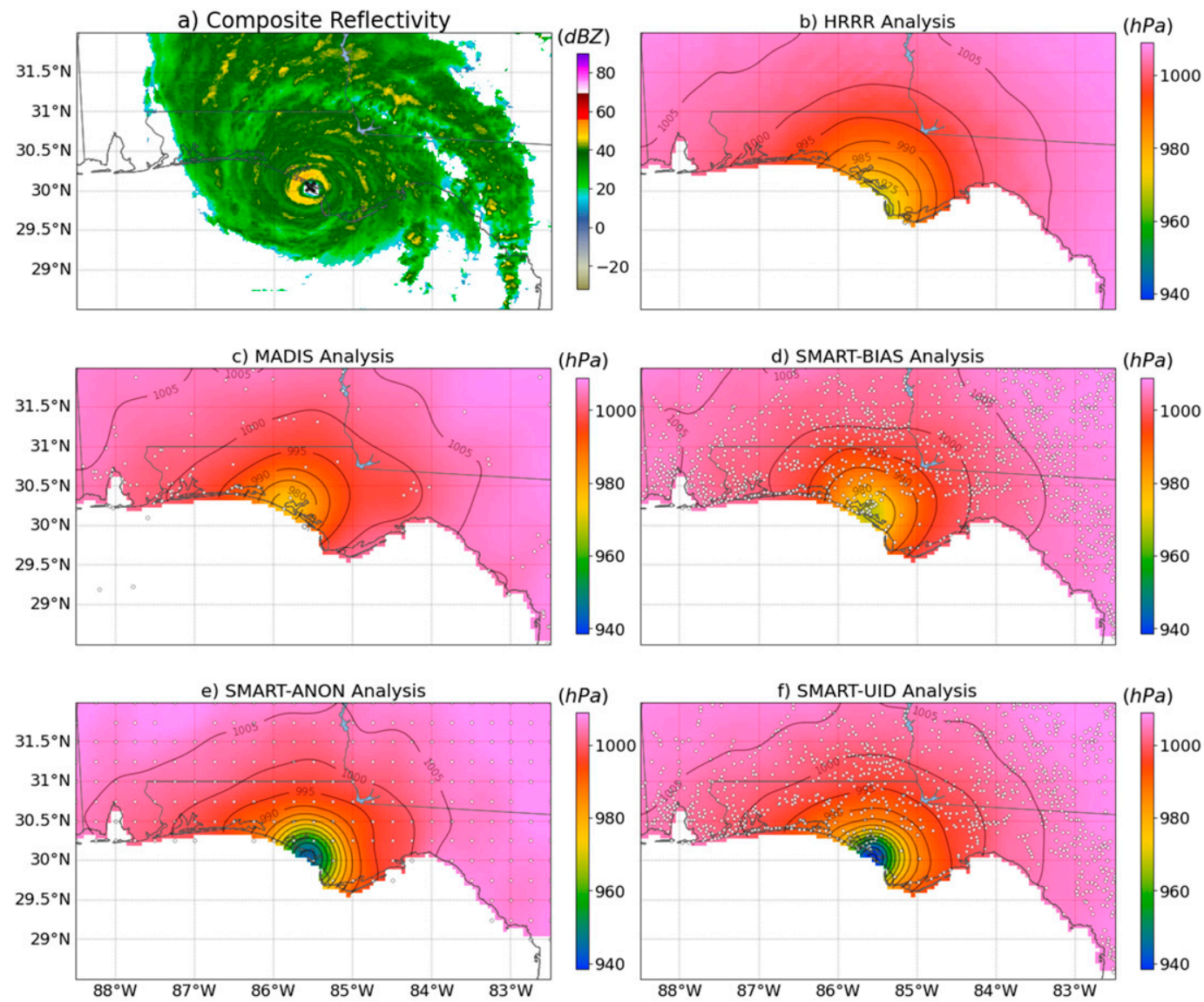

g) $12-\mathrm{h}$ Pressure Time Series at $\operatorname{KPAM}\left(30.06^{\circ} \mathrm{N}, 85.57^{\circ} \mathrm{W}\right)$

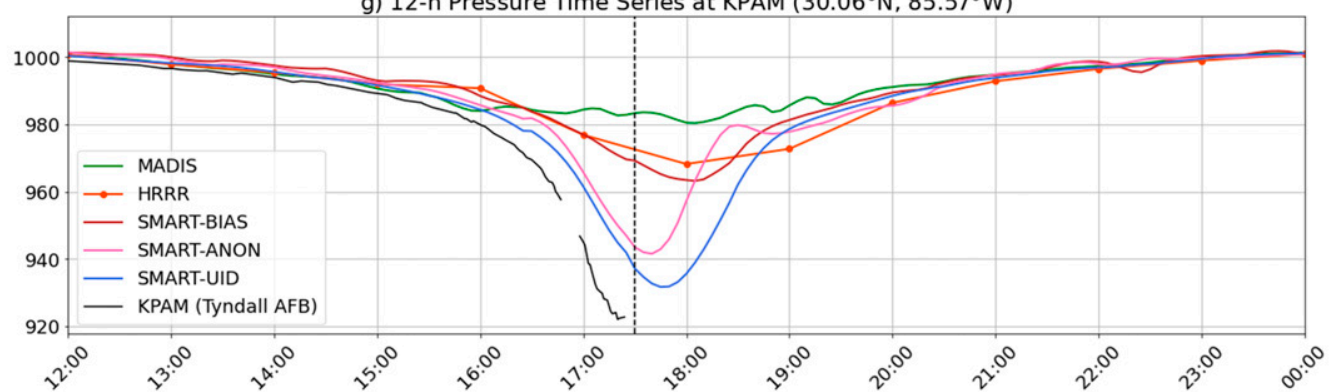

FIG. 12. (a) Composite reflectivity and (b) HRRR, (c) MADIS, and (d)-(f) smartphone pressure analyses during the landfall of Hurricane Michael at 1730 UTC 18 Sep 2018. In (a), an X marker highlights the location for which gridded pressure time series were extracted from pressure analyses, for comparison with an observed pressure trace at KPAM. (g) 12-h pressure time series from 5-min pressure analyses were compared to the observed pressure trace at KPAM (Tyndall AFB) during the landfall of Hurricane Michael. Gray (circular) markers denote locations of pressure observations contributing to pressure analyses. Isobars are contoured in black at intervals of $5 \mathrm{hPa}$.

$43 \%$ and $57 \%$, respectively. While no SPOs were removed during bias correction, $33 \%$ of UID SPOs and $3 \%$ of ANON SPOs were removed during QC. After bias correction and QC, domain-average UID (ANON) SPO errors fell by $85 \%(43 \%)$.

To examine whether SPOs can provide better pressure analyses than current surface pressure networks, multiresolution kriging was used to generate 5-min smartphone pressure analyses. These analyses were verified using high-quality METAR observations from (5 min) ASOS and (20 min) AWOS sites. The results revealed that ANON and UID SPOs produced smartphone pressure analyses that outperformed MADIS by $21 \%$ and $31 \%$, respectively, suggesting that the quality of smartphone pressure analyses was not substantially reduced by anonymization. At high-frequency ASOS verification sites, both SMART-ANON and SMART-UID analyses outperformed MADIS. This was 

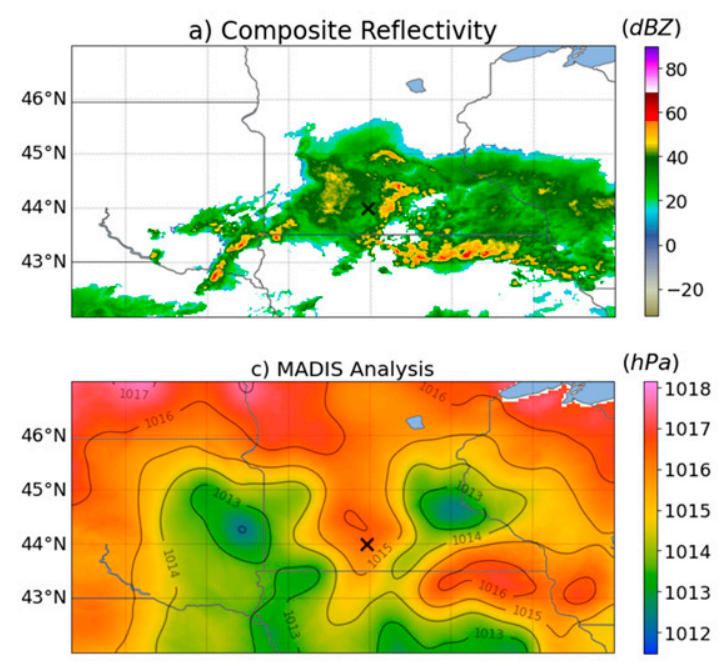

$(\mathrm{hPa})$
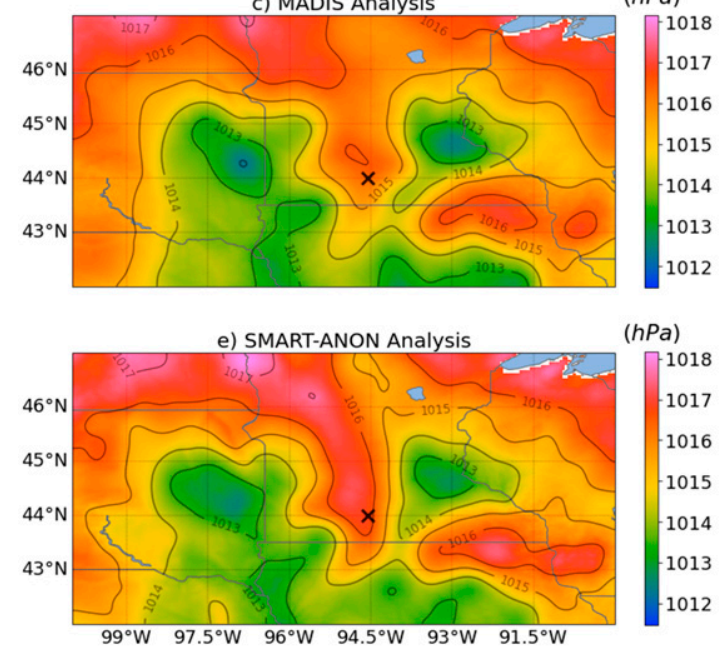
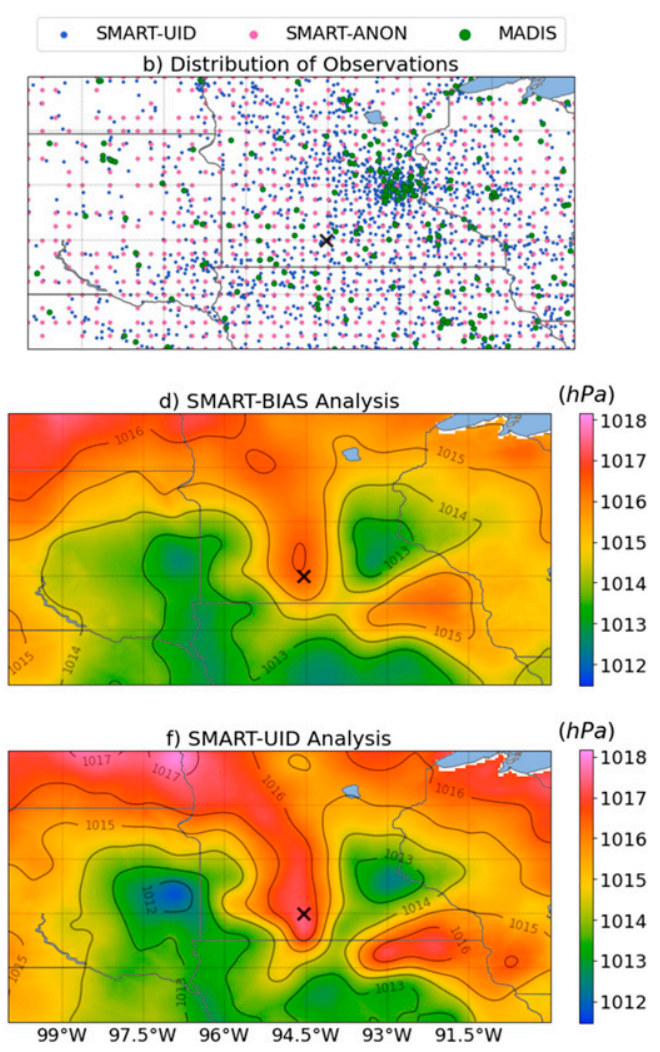

YG $\left(43.99^{\circ} \mathrm{N}, 94.56^{\circ} \mathrm{W}\right)$

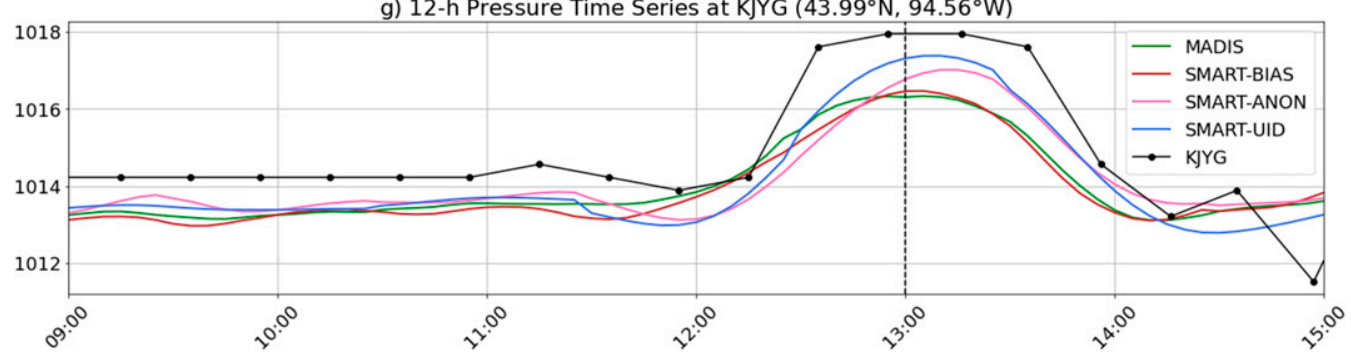

FIG. 13. Analysis of an upper-Midwest MCS at 1300 UTC 18 Sep 2018. (a) The MCS is visualized in the composite reflectivity analysis. (b) The distribution of MADIS observations and SPOs, during the 5-min analysis window around 1300 UTC. Corrected UID (ANON) SPOs contribute to SMART-UID (ANON) analyses and are labeled as such in (b). (c)-(f) MADIS and smartphone pressure analyses. Isobars are contoured in black in intervals of 1 $\mathrm{hPa}$. A black X marker in (a)-(f) highlights the location at which pressure time series were retrieved, for comparison with a METAR pressure trace from KYJG. (g) 12-h pressure time series, from smartphone/MADIS pressure analyses, were compared to the observed pressure (altimeter) trace at KYJG.

especially true in regions of the analysis domain where MADIS observations were less dense.

To assess whether SPOs could better capture mesoscale phenomena poorly resolved by existing surface pressure networks, smartphone pressure analyses were compared to MADIS pressure analyses for two high-impact weather events. For the case of Hurricane Michael, smartphone pressure analyses better captured the gradient and central pressure associated with the landfall than MADIS. Specifically, the high spatiotemporal density of SPOs ensured that the tight pressure minimum of Michael was defined, while MADIS pressure analyses poorly resolved the low pressure center due to the sparsity of MADIS pressure data along the storm path. During the second event in which a long-lived MCS passed through the upper Midwest, both SMART-ANON and SMART-UID analyses showed a distinct mesohigh flanked by areas of lower pressure. The spatial characteristics of mesoscale pressure features in SMART-ANON and SMART-UID analyses were remarkably consistent, suggesting that, for large mesoscale pressure features, little information is lost when SPOs are retrieved anonymously. While both SMART-BIAS and MADIS analyses observed a region of higher pressure associated with the MCS, both analyses 


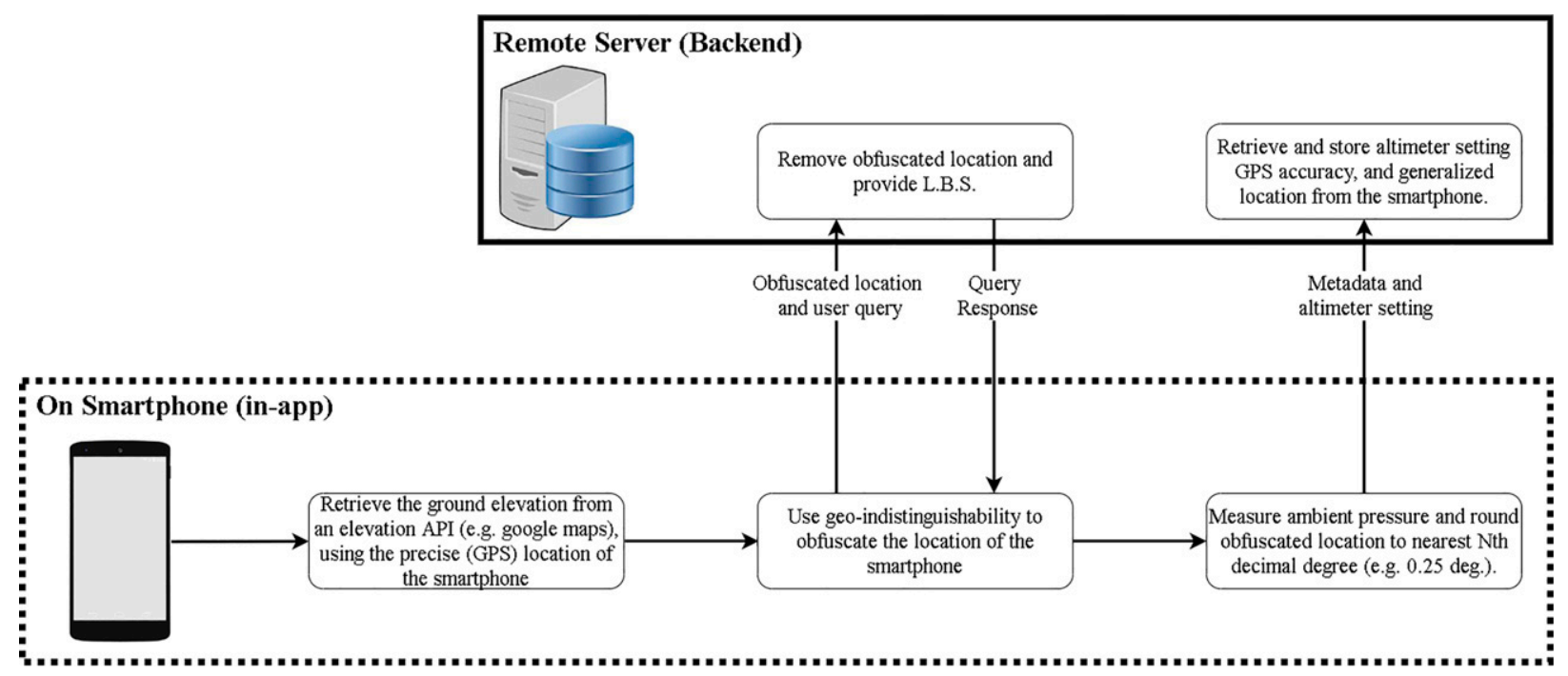

FIG. A1. Flowchart depicting a decentralized framework for anonymous crowdsourcing of smartphone pressure observations. This framework can be incorporated into the workflow of any app that provides a location-based service (LBS). Note that as of July 2020, compliance with the GDPR requires a data processor agreement to retrieve elevation from a third-party application programming interface (API; e.g., Google). Fortunately, this issue could be mitigated by setting up a first-party elevation API to avoid sharing location data with third parties.

underestimated the pressure gradient across the storm and the amplitude of the mesohigh.

This study demonstrates the potential for SPOs to enhance mesoscale pressure analyses and augment surface pressure networks in regions where existing observations are sparse. To date, legal and technological issues have hindered the use of SPOs in NWP. To resolve these issues, an approach was developed for anonymizing and bias correcting billions of SPOs. Importantly, anonymizing SPOs did not substantially degrade the performance of smartphone pressure analyses. This finding suggests that necessary privacy protections need not compromise the potential of SPOs for NWP. Adopting an anonymous crowdsourcing framework for SPOs should encourage wider adoption of smartphone pressure retrieval, greater collaboration, and improved forecasts.

Acknowledgments. The authors acknowledge IBM (the Weather Company) for their financial support of this research and their provision of pressure data. Peter Neilley of the Weather Company was instrumental in the acquisition of IBM smartphone pressure data.

Data availability statement. Due to privacy and ethical concerns, as well as confidentiality agreements and data storage limitations, the smartphone pressure observations used in this study cannot be made publicly available. Conventional surface pressure observations from ASOS stations were retrieved from the National Climatic Data Center (NCEI 2018) for the following website: https://www.ncdc.noaa.gov/data-access/ land-based-station-data/land-based-datasets/automated-surfaceobserving-system-asos. METAR and meteorological surface (i.e., mesonet) observations from MADIS are available through a request from https://madis.ncep.noaa.gov/data_application.shtml.
In this study, surface pressure observations from restricted mesonets were used. These observations are not publicly accessible and are only available to researchers (see https:// madis.ncep.noaa.gov/madis_restrictions.shtml). Source code and sample data have been made available through the opensource repository meteo-krig (https:/github.com/cmac994/ meteo-krig), which illustrates the generation of pressureanalyses from publicly available MADIS observations. A demonstration of the anonymization methodology, presented in section 3, has also been made available (https://github.com/ cmac994/spo_anon).

\section{APPENDIX A}

\section{Decentralized Anonymization of SPOs}

To date, the obfuscation and generalization of SPO location data anonymize SPO data after retrieval, requiring the collection and storage of personally identifiable location data. Ultimately, SPO anonymization should be performed on the user's device before retrieval. Figure A1 illustrates a decentralized framework for SPO anonymization that can be incorporated into the workflow of any mobile application providing location-based services. The novelty of this decentralized approach is that it ensures personally identifiable information (e.g., latitude, longitude, and elevation) is never stored outside the user's device. In this framework, user locations are obfuscated on the device and submitted to a remote server. In the process of ensuring geo-indistinguishability, obfuscated locations are typically within a kilometer of the user's real location; therefore, location obfuscation should not have a detrimental impact on most applications (Micinski et al. 2013). Since the geo-indistinguishable location is not stored remotely, the user's 
location is afforded some protection from observation attacks (Primault et al. 2014). Upon completion of a query, the location is further obscured through rounding and then submitted to a remote server as an anonymous SPO.

In this approach, bandwidth usage would not be a concern since many apps already collect user location data to provide a location-based service. Experiments from McNicholas and Mass (2018a) showed that SPOs could be retrieved as frequently as every $15 \mathrm{~min}$ without substantially impacting battery life or bandwidth constraints. Furthermore, the decentralized framework for SPO anonymization would not affect the calculation of privacy metrics, which depend on population data and the total number of users contributing SPOs (appendix B).

\section{APPENDIX B}

\section{Estimation of $K$-Anonymity}

During anonymization, geo-indistinguishable SPOs are generalized to a temporal resolution of $5 \mathrm{~min}$ and a spatial resolution of $0.25^{\circ}$. The expected level of $k$-anonymity is estimated by counting the number of people who may have contributed SPOs to the dataset within each $0.25^{\circ}$ grid box during a 5-min observation window. This is estimate is calculated using the following equation:

$$
k=P_{s} \times \max \left[P, \mathrm{AADT} \times\left(T_{H} / 12\right)\right],
$$

where $P_{s}$ is the fraction of the population contributing SPOs, $P$ is the fixed population count within the $0.25^{\circ}$ grid box (Columbia University 2018), AADT is the maximum annual average daily traffic count within the grid box (Federal Highway Administration 2017), and $T_{H}$ is the percentage of daily traffic by hour (U.S. Department of Transportation 2017). This approach expands upon efforts to estimate $k$-anonymity for public health datasets using local population density (Cassa et al. 2006; Allshouse et al. 2010). In sparsely populated regions, the number of people passing through a $0.25^{\circ}$ grid box can exceed the number of permanent residents within the grid box. Consequently, the maximum value of population count and 5-min vehicular traffic is computed. To estimate $k$, the resulting count is multiplied by the fraction of the population contributing SPOs. This estimate of $k$-anonymity thus assumes that a potential adversary has prior knowledge of who participates in the network (i.e., who contributes SPOs). In Fig. 4a, the minimum $k$-anonymity for SPOs within $0.25^{\circ}$ grid boxes was computed by calculating the minimum $k$-anonymity over a $24-\mathrm{h}$ period. The $k$-anonymity is a useful privacy metric since laws governing personally identifiable data [e.g., General Data Protection Regulation (GDPR), California Consumer Privacy Act (CCPA)] utilize a risk-based approach to determine whether data is personal thus subject to regulation (Finck and Pallas 2020).

\section{REFERENCES}

Alexander, C., and Coauthors, 2017: WRF-ARW research to operations update: The Rapid-Refresh (RAP) version 4, HighResolution Rapid Refresh (HRRR) version 3 and convectionallowing ensemble prediction. 18th WRF User's Workshop, Boulder, CO, UCAR-NCAR, 2.5, https://ruc.noaa.gov/ruc/ppt_ pres/Alexander_WRFworkshop_2017_Final.pdf.
Allshouse, W. B., M. K. Fitch, K. H. Hampton, D. C. Gesink, I. A. Doherty, P. A. Leone, M. L. Serre, and W. C. Miller, 2010: Geomasking sensitive health data and privacy protection: An evaluation using an E911 database. Geocarto Int., 25, 443-452, https://doi.org/10.1080/10106049.2010.496496.

Andrés, M. E., N. E. Bordenabe, K. Chatzikokolakis, and C. Palamidessi, 2013: Geo-indistinguishability: Differential privacy for location-based systems. Proc. 2013 ACM SIGSAC Conf. on Computer \& Communications Security, New York, NY, Association for Computing Machinery, 901-914, https:// doi.org/10.1145/2508859.2516735.

Arthur, R., C. A. Boulton, H. Shotton, and H. T. P. Williams, 2018: Social sensing of floods in the UK. PLOS ONE, 13, e0189327, https://doi.org/10.1371/journal.pone.0189327.

Bamba, B., L. Liu, P. Pesti, and T. Wang, 2008: Supporting anonymous location queries in mobile environments with PrivacyGrid. Proc. 17th Int. Conf. on World Wide Web, New York, NY, Association for Computing Machinery, 237-246, https://doi.org/ 10.1145/1367497.1367531.

Bauer, P., A. Thorpe, and G. Brunet, 2015: The quiet revolution of numerical weather prediction. Nature, 525, 47-55, https:// doi.org/10.1038/nature14956.

Benjamin, S. G., B. D. Jamison, W. R. Moninger, S. R. Sahm, B. E. Schwartz, and T. W. Schlatter, 2010: Relative short-range forecast impact from aircraft, profiler, radiosonde, VAD, GPS-PW, METAR, and mesonet observations via the RUC hourly assimilation cycle. Mon. Wea. Rev., 138, 1319-1343, https://doi.org/10.1175/2009MWR3097.1.

, J. M. Brown, G. Brunet, P. Lynch, K. Saito, and T. W. Schlatter, 2019: 100 years of progress in forecasting and NWP applications. A Century of Progress in Atmospheric and Related Sciences: Celebrating the American Meteorological Society Centennial, Meteor. Monogr., No. 59, Amer. Meteor. Soc., https://doi.org/10.1175/AMSMONOGRAPHS-D-18-0020.1.

Blaylock, B., J. Horel, and S. Liston, 2017: Cloud archiving and data mining of High-Resolution Rapid Refresh forecast model output. Comput. Geosci., 109, 43-50, https://doi.org/10.1016/ j.cageo.2017.08.005.

Bordenabe, N. E., K. Chatzikokolakis, and C. Palamidessi, 2014: Optimal geo-indistinguishable mechanisms for location privacy. Proc. 2014 ACM SIGSAC Conf. on Computer and Communications Security, New York, NY, Association for Computing Machinery, 251-262, https://doi.org/10.1145/ 2660267.2660345 .

Boukoros, S., M. Humbert, S. Katzenbeisser, and C. Troncoso, 2019: On (the lack of) location privacy in crowdsourcing applications. Proc. 28th USENIX Conf. on Security Symposium, Santa Clara, CA, USENIX Association, 1859-1876, https:// www.usenix.org/system/files/sec19-boukoros.pdf.

Cassa, C. A., S. J. Grannis, J. M. Overhage, and K. D. Mandl, 2006: A context-sensitive approach to anonymizing spatial surveillance data: Impact on outbreak detection. J. Amer. Med. Inf. Assoc., 13, 160-165, https://doi.org/10.1197/jamia.M1920.

Chang, W., D. Jacques, L. Fillion, and S. Baek, 2017: Assimilation of hourly surface observations with the Canadian highresolution ensemble Kalman filter. Atmos.-Ocean, 55, 247263, https://doi.org/10.1080/07055900.2017.1384361.

Chatzikokolakis, K., C. Palamidessi, and M. Stronati, 2015: Geoindistinguishability: A principled approach to location privacy. Distributed Computing and Internet Technology: ICDCIT 2015, R. Natarajan, G. Barua, and M. R. Patra, Eds., Lecture Notes in Computer Science, Vol. 8956, Springer, 49-72, https://doi.org/ 10.1007/978-3-319-14977-6_4. 
Chen, T., and C. Guestrin, 2016: XGBoost: A scalable tree boosting system. Proc. 22nd ACM SIGKDD Int. Conf. on Knowledge Discovery and Data Mining, New York, NY, Association for Computing Machinery, 785-794, https:// doi.org/10.1145/2939672.2939785.

Clark, M. R., J. D. C. Webb, and P. J. Kirk, 2018: Fine-scale analysis of a severe hailstorm using crowd-sourced and conventional observations. Meteor. Appl., 25, 472-492, https://doi.org/10.1002/met.1715.

COESA, 1976: U.S. Standard Atmosphere, 1976. NOAA, 227 pp., https:/www.ngdc.noaa.gov/stp/space-weather/onlinepublications/miscellaneous/us-standard-atmosphere-1976/ us-standard-atmosphere_st76-1562_noaa.pdf.

Columbia University, 2018: Gridded Population of the World, version 4 (GPWv4): Population count adjusted to Match 2015 revision of UN WPP country totals, revision 11. NASA Socioeconomic Data and Applications Center, Center for International Earth Science Information Network, accessed 23 November 2020, https://doi.org/10.7927/H4PN93PB.

Compo, G. P., J. S. Whitaker, and P. D. Sardeshmukh, 2006: Feasibility of a 100-year reanalysis using only surface pressure data. Bull. Amer. Meteor. Soc., 87, 175-190, https://doi.org/ 10.1175/BAMS-87-2-175.

De Montjoye, Y. A., C. Hidalgo, M. Verleysen, and V. D. Blondel, 2013: Unique in the crowd: The privacy bounds of human mobility. Sci. Rep., 3, 1376, https://doi.org/10.1038/srep01376.

Dirren, S., R. Torn, and G. Hakim, 2007: A data assimilation case study using a limited-area ensemble filter. Mon. Wea. Rev., 135, 1455-1473, https://doi.org/10.1175/MWR3358.1.

Droste, M. A., B. G. Heusinkveld, D. Fenner, and G. Steenveld, 2020: Assessing the potential and application of crowdsourced urban wind data. Quart. J. Roy. Meteor. Soc., 146, 2671-2688, https://doi.org/10.1002/qj.3811.

Ericsson, 2020: Regional subscriptions outlook. Ericsson mobility report, 12-13, accessed 23 November 2020, https:// www.ericsson.com/49da93/assets/local/mobility-report/documents/ 2020/june2020-ericsson-mobility-report.pdf.

Ester, M., H.-P. Kriegel, J. Sander, and X. Xu, 1996: A density-based algorithm for discovering clusters in large spatial databases with noise. Proc. Second Int. Conf. on Knowledge Discovery and Data Mining, Portland, OR, AAAI, 226-231, https://www.aaai.org/ Papers/KDD/1996/KDD96-037.pdf.

European Union, 2018: Regulation (EU) 2016/679 of the European parliament and of the council. Official Journal of the European Union, 88 pp., accessed 23 November 2020, https://eur-lex.europa.eu/ legal-content/EN/TXT/PDF/?uri=CELEX:32016R0679.

Federal Highway Administration, 2017: HPMS public release of geospatial data in shapefile format. Office of Highway Policy Information, accessed 23 November 2020, https:// www.fhwa.dot.gov/policyinformation/hpms/shapefiles_2017.cfm.

Finck, M., and F. Pallas, 2020: They who must not be identified-Distinguishing personal from non-personal data under the GDPR. Int. Data Privacy Law, 10, 11-36, https://doi.org/10.1093/idpl/ipz026.

Fung, B. C. M., K. Wang, R. Chen, and P. S. Yu, 2010: Privacypreserving data publishing: A survey of recent developments. ACM Comput. Surv., 42, 14, https://doi.org/10.1145/ 1749603.1749605.

Gasperoni, N. A., X. Wang, K. A. Brewster, and F. H. Carr, 2018: Assessing impacts of the high-frequency assimilation of surface observations for the forecast of convection initiation on 3 April 2014 within the Dallas-Fort Worth test bed. Mon. Wea. Rev., 146, 3845-3872, https://doi.org/10.1175/MWR-D18-0177.1.
Gesch, D. B., M. J. Oimoen, and G. A. Evans, 2014: Accuracy assessment of the U.S. Geological Survey National Elevation Dataset, and comparison with other large-area elevation datasets-SRTM and ASTER. U.S. Geological Survey OpenFile Rep. 2014-1008, 10 pp., https://pubs.usgs.gov/of/2014/ 1008/pdf/ofr2014-1008.pdf.

Gruteser, M., and D. Grunwald, 2003: Anonymous usage of location-based services through spatial and temporal cloaking. Proc. ACM Int. Conf. Mobile Systems Applications and Services, San Francisco, CA, USENIX Association, 31-42, https://dl.acm.org/doi/pdf/10.1145/1066116.1189037.

Gustafsson, N., and Coauthors, 2018: Survey of data assimilation methods for convective-scale numerical weather prediction at operational centres. Quart. J. Roy. Meteor. Soc., 144, 12181256, https://doi.org/10.1002/qj.3179.

Haiden, T., and Coauthors, 2018: Evaluation of ECMWF forecasts, including the 2018 upgrade. ECMWF Tech. Memo. 834, 26 pp., https://www.ecmwf.int/sites/default/files/elibrary/2018/18748use-situ-surface-observations-ecmwf.pdf.

Hintz, K. S., H. Vedel, and E. Kaas, 2019a: Collecting and processing of barometric data from smartphones for potential use in numerical weather prediction data assimilation. Meteor. Appl., 26, 733-746, https://doi.org/10.1002/met.1805.

— , and Coauthors, 2019b: Collecting and utilizing crowdsourced data for numerical weather prediction: Propositions from the meeting held in Copenhagen, 4-5 December 2018. Atmos. Sci. Lett., 20, e921, https://doi.org/10.1002/asl.921.

Horel, J., and Coauthors, 2002: Mesowest: Cooperative mesonets in the western United States. Bull. Amer. Meteor. Soc., 83, 211-225, https://doi.org/10.1175/1520-0477(2002)083<0211: MCMITW $>2.3 . \mathrm{CO} ; 2$.

Ingleby, B., 2015: Global assimilation of air temperature, humidity, wind and pressure from surface stations. Quart. J. Roy. Meteor. Soc., 141, 504-517, https://doi.org/10.1002/qj.2372.

Jacques, A. A., J. D. Horel, E. T. Crosman, and F. L. Vernon, 2015: Central and eastern U.S. surface pressure variations derived from the USArray network. Mon. Wea. Rev., 143, 1472-1493, https://doi.org/10.1175/MWR-D-14-00274.1.

Kim, N.-Y., Y.-H. Kim, Y. Yoon, H.-H. lm, R. K. Y. Choi, and Y. H. Lee, 2015: Correcting air-pressure data collected by MEMS sensors in smartphones. J. Sens., 2015, 245498, https:// doi.org/10.1155/2015/245498.

Kim, Y.-H., J.-H. Ha, Y. Yoon, N.-Y. Kim, H.-H. Im, S. Sim, and R. K. Y. Choi, 2016: Improved correction of atmospheric pressure data obtained by smartphones through machine learning. Comput. Intell. Neurosci., 2016, 9467878, https:// doi.org/10.1155/2016/9467878.

Kraemer, M. U. G., and Coauthors, 2020: Mapping global variation in human mobility. Nat. Hum. Behav., 4, 800-810, https:// doi.org/10.1038/s41562-020-0875-0.

Krumm, J., 2007: Inference attacks on location tracks. Pervasive Computing: Pervasive 2007, A. LaMarca, M. Langheinrich, and K. N. Truong, Eds., Lecture Notes in Computer Science, Vol. 4480, Springer, https://doi.org/10.1007/978-3-540-720379_8143, https://doi.org/10.1145/2508859.2516735.

Leech, J., W. Nelson, R. Burnett, S. Aaron, and M. E. Raizenne, 2002: It's about time: A comparison of Canadian and American time-activity patterns. J. Expo. Sci. Environ. Epidemiol., 12, 427-432, https://doi.org/10.1038/sj.jea.7500244.

Li, L., and M. F. Goodchild, 2013: Is privacy still an issue in the era of big data-Location disclosure in spatial footprints. 21st Int. Conf. on Geoinformatics, Kaifeng, China, IEEE, 1-4, https:// doi.org/10.1109/Geoinformatics.2013.6626191. 
Li, R., Q. Zhang, J. Sun, Y. Chen, L. Ding, and T. Wang, 2021: Smartphone pressure data: Quality control and impact on atmospheric analysis. Atmos. Meas. Tech., 14, 785-801, https:// doi.org/10.5194/amt-14-785-2021.

Liu, Z. Q., and F. Rabier, 2002: The interaction between model resolution, observation resolution and observation density in data assimilation: A one-dimensional study. Quart. J. Roy. Meteor. Soc., 128, 1367-1386, https://doi.org/10.1256/ 003590002320373337.

Madaus, L. E., and C. F. Mass, 2017: Evaluating smartphone pressure observations for mesoscale analyses and forecasts. Wea. Forecasting, 32, 511-531, https://doi.org/10.1175/WAFD-16-0135.1.

— , G. J. Hakim, and C. F. Mass, 2014: Utility of dense pressure observations for improving mesoscale analyses and forecasts. Mon. Wea. Rev., 142, 2398-2413, https://doi.org/10.1175/MWRD-13-00269.1.

Mandement, M., and O. Caumont, 2020: Contribution of personal weather stations to the observation of deep-convection features near the ground. Nat. Hazards Earth Syst. Sci., 20, 299322, https://doi.org/10.5194/nhess-20-299-2020.

Mass, C., and L. Madaus, 2014: Surface pressure observations from smartphones: A potential revolution for high-resolution weather prediction. Bull. Amer. Meteor. Soc., 95, 1343-1349, https://doi.org/10.1175/BAMS-D-13-00188.1.

McNicholas, C., and C. F. Mass, 2018a: Smartphone pressure collection and bias correction using machine learning. J. Atmos. Oceanic Technol., 35, 523-540, https://doi.org/10.1175/JTECHD-17-0096.1.

— observations on forecast skill during two case studies in the Pacific Northwest. Wea. Forecasting, 33, 1375-1396, https:// doi.org/10.1175/WAF-D-18-0085.1.

Meier, F., D. Fenner, T. Grassmann, M. Otto, and D. Scherer, 2017: Crowdsourcing air temperature from citizen weather stations for urban climate research. Urban Climate, 19, 170-191, https://doi.org/10.1016/j.uclim.2017.01.006.

Mendes, R., M. Cunha, and J. P. Vilela, 2020: Impact of frequency of location reports on the privacy level of geo-indistinguishability. Proc. Privacy Enhancing Technol., 2020, 379-396, https://doi.org/10.2478/popets-2020-0032.

Micinski, K., P. Phelps, and J. S. Foster, 2013: An empirical study of location truncation on Android. Mobile Security Technologies (MOST) 2013, San Francisco, CA, IEEE and IACR, https:// www.cs.tufts.edu/ jfoster/papers/most13.pdf.

Miller, P. A., and S. G. Benjamin, 1992: A system for the hourly assimilation of surface observations in mountainous and flat terrain. Mon. Wea. Rev., 120, 2342-2359, https://doi.org/ 10.1175/1520-0493(1992)120<2342:ASFTHA > 2.0.CO;2.

—_, M. F. Barth, and L. A. Benjamin, 2005: An update on MADIS observation ingest, integration, quality control and distribution capabilities. 21st Int. Conf. on Interactive Information and Processing Systems for Meteorology, Oceanography, and Hydrology, San Diego, CA, Amer. Meteor. Soc., J7.12, https://ams.confex.com/ams/pdfpapers/ 86703.pdf.

Muller, C. L., L. Chapman, S. Johnston, C. Kidd, S. Illingworth, G. Foody, A. Overeem, and R. R. Leigh, 2015: Crowdsourcing for climate and atmospheric sciences: Current status and future potential. Int. J. Climatol., 35, 3185-3203, https://doi.org/ $10.1002 /$ joc. 4210 .

Napoly, A., T. Grassmann, F. Meier, and D. Fenner, 2018: Development and application of a statistically-based quality control for crowdsourced air temperature data. Front. Earth Sci., 6, 118, https://doi.org/10.3389/feart.2018.00118.

NCEI, 2018: 1-minute Automated Surface Observing System (ASOS) data. NOAA, accessed 23 November 2020, https:// www.ncdc.noaa.gov/data-access/land-based-station-data/landbased-datasets/automated-surface-observing-system-asos.

NCEP, 2017: MADIS meteorological surface quality control checks. NOAA, accessed 4 March 2021, https://madis.ncep.noaa.gov/ madis_sfc_qc_notes.shtml.

Nipen, T. N., I. A. Seierstad, C. Lussana, J. Kristiansen, and $\varnothing$. Hov, 2020: Adopting citizen observations in operational weather prediction. Bull. Amer. Meteor. Soc., 101, E43-E57, https://doi.org/10.1175/BAMS-D-18-0237.1.

Niu, B., Y. Chen, Z. Wang, F. Li, B. Wang, and H. Li, 2021: Eclipse: Preserving differential location privacy against long-term observation attacks. IEEE Trans. Mobile Comput., https:// doi.org/10.1109/TMC.2020.3000730, in press.

Nychka, D., S. Bandyopadhyay, D. Hammerling, F. Lindgren, and S. Sain, 2015: A multiresolution Gaussian process model for the analysis of large spatial datasets. J. Comput. Graph. Stat., 24, 579-599, https://doi.org/10.1080/10618600.2014.914946.

, D. Hammerling, S. Sain, and N. Lenssen, 2016: LatticeKrig: Multiresolution kriging based on Markov random fields, version 8.4. R package, https://doi.org/10.5065/D6HD7T1R.

Primault, V., S. B. Mokhtar, C. Lauradoux, and L. Brunie, 2014: Differentially private location privacy in practice. Third Workshop on Mobile Security Technologies (MoST), San Jose, CA, IEEE, https://hal.archives-ouvertes.fr/hal01148230.

Rabier, F., P. Gauthier, and R. Langland, 2007: Objectives of the THORPEX working group on data assimilation and observing strategies for high impact weather forecast improvements. Geophysical Research Abstracts, Vol. 9, Abstract 04024, https:// meetings.copernicus.org/www.cosis.net/abstracts/EGU2007/04024/ EGU2007-J-04024.pdf.

Samarati, P., and L. Sweeny, 1998: Protecting privacy when disclosing information: $k$-anonymity and its enforcement through generalization and suppression. SRI Computer Science Laboratory Tech. Rep. SRI-CSL-98-04, 19 pp., https://epic.org/privacy/ reidentification/Samarati_Sweeney_paper.pdf.

Simmons, A., and A. Hollingsworth, 2002: Some aspects of the improvement in skill of numerical weather prediction. Quart. J. Roy. Meteor. Soc., 128, 647-677, https://doi.org/10.1256/ 003590002321042135.

Sobash, R. A., and D. J. Stensrud, 2015: Assimilating surface mesonet observations with the EnKF to improve ensemble forecasts of convection initiation on 29 May 2012. Mon. Wea. Rev., 143, 3700-3725, https://doi.org/10.1175/MWR-D-14-00126.1.

State of California, 2020: California consumer privacy act. 28 pp., accessed 23 November 2020, https://oag.ca.gov/sites/all/files/ agweb/pdfs/privacy/oal-sub-final-text-of-regs.pdf.

Sun, J., and Coauthors, 2014: Use of NWP for nowcasting convective precipitation: Recent progress and challenges. Bull. Amer. Meteor. Soc., 95, 409-426, https://doi.org/10.1175/BAMS-D-1100263.1 .

Tyndall, D. P., and J. D. Horel, 2013: Impacts of mesonet observations on meteorological surface analyses. Wea. Forecasting, 28, 254-269, https://doi.org/10.1175/WAF-D-12-00027.1.

U.S. Department of Transportation, 2017: National Household Travel Survey. Accessed 23 November 2020, https://nhts.ornl.gov/.

Wadler, J. B., J. A. Zhang, R. F. Rogers, B. Jaimes, and L. K. Shay, 2021: The rapid intensification of Hurricane Michael (2018): Storm structure and the relationship to environmental and 
air-sea interactions. Mon. Wea. Rev., 149, 245-267, https:// doi.org/10.1175/MWR-D-20-0145.1.

Wang, J., and M. P. Kwan, 2020: Daily activity locations $k$-anonymity for the evaluation of disclosure risk of individual GPS datasets. Int. J. Health Geogr., 19, 7, https://doi.org/10.1186/s12942020-00201-9.

Wheatley, D. M., and D. J. Stensrud, 2010: The impact of assimilating surface pressure observations on severe weather events in a WRF mesoscale ensemble system. Mon. Wea. Rev., 138, 1673-1694, https://doi.org/10.1175/2009MWR3042.1.
WMO, 2014: Integrated African strategy on meteorology. AMCOMET, 37 pp., https://amcomet.wmo.int/en/pages/integrated-africanstrategy-meteorology-weather-and-climate-services.

Zandbergen, P. A., 2014: Ensuring confidentiality of geocoded health data: Assessing geographic masking strategies for individual-level data. Adv. Med., 2014, 567049, https://doi.org/ $10.1155 / 2014 / 567049$.

Zheng, F., and Coauthors, 2018: Crowdsourcing methods for data collection in geophysics: State of the art, issues, and future directions. Rev. Geophys., 56, 698-740, https://doi.org/10.1029/2018RG000616. 